\title{
STABILITY OF SUBMANIFOLDS WITH PARALLEL MEAN CURVATURE IN CALIBRATED MANIFOLDS
}

\author{
Isabel M.C. Salavessa \\ Centro de Física das Interacções Fundamentais, Instituto Superior Técnico, Technical University of Lis- \\ bon, Edifício Ciência, Piso 3, Av. Rovisco Pais, 1049-001 Lisboa, Portugal; isabel.salavessa@ist.utl.pt
}

\begin{abstract}
On a Riemannian manifold $\bar{M}^{m+n}$ with an $(m+1)$-calibration $\Omega$, we prove that an $m$ submanifold $M$ with constant mean curvature $H$ and calibrated extended tangent space $\mathbb{R} H \oplus T M$ is a critical point of the area functional for variations that preserve the enclosed $\Omega$-volume. This recovers the case described by Barbosa, do Carmo and Eschenburg, when $n=1$ and $\Omega$ is the volume element of $\bar{M}$. To the second variation we associate an $\Omega$-Jacobi operator and define $\Omega$-stability. Under natural conditions, we show that the Euclidean $m$-spheres are the unique $\Omega$ stable submanifolds of $\mathbb{R}^{m+n}$. We study the $\Omega$-stability of geodesic $m$-spheres of a fibred space form $M^{m+n}$ with totally geodesic $(m+1)$-dimensional fibres.
\end{abstract}

\section{Introduction}

Immersed hypersurfaces with constant mean curvature of a Euclidean space are known to be critical points of a variational problem, namely, they are critical points of the $m$ area $A_{D}(t)$ for all variations $\phi_{t}: D \subset M^{m} \rightarrow \mathbb{R}^{m+1}$ of $\phi=\phi_{0}$ fixing the boundary of a compact domain $D$, and that leave a certain enclosed $(m+1)$-volume $V_{D}(t)$ invariant. This volume can be given by $V_{D}(\phi)=\frac{1}{m+1} \int_{D}\langle\phi, v\rangle d M$, where $v$ is the unit normal to $\phi$, and its modulo is the volume of the cone over $\phi(D)$ with vertex at $0 \in \mathbb{R}$ (see [2]). This property was generalized by Barbosa, do Carmo and Eschenburg in [3] to hypersurfaces with constant mean curvature $H$ immersed in a Riemannian manifold $\bar{M}^{m+1}$, by defining the volume of a variation $\bar{\phi}(t, p)=\phi_{t}(p)$ as $V_{D}(t)=\int_{[0, t] \times D} \bar{\phi}^{*} d \bar{M}$. A critical point of $A_{D}(t)$ for volume-preserving variations, i.e., $V_{D}(t)=V_{D}(0)=0$, is just a critical point of $J_{D}(t)=A_{D}(t)+m H_{0} V_{D}(t)$ for any variation fixing the boundary, where $H_{0}$ is the mean value of the mean curvature $H$ of $\phi$, and it is characterized by having constant mean curvature $H_{0}$. Such a critical point is stable if $A_{D}^{\prime \prime}(0) \geq 0$ for all volume preserving variations, or equivalently, if $J_{D}^{\prime \prime}(0) \geq 0$ for all variations with vector variation $W$ satisfying

MSC 2000: Primary: 53C42; 53C38. Secondary: 58E12; 35J19; 47A75

Key Words: Stability, Parallel Mean curvature, Isoperimetric Problem, Calibration.

Partially supported by FCT through program PTDC/MAT/101007/2008. 
$W^{\perp}=f v$, where $f \in \mathscr{F}_{D}$ and $v$ is the unit normal of $M$. The class $\mathscr{F}_{D}$ is given by the functions $f: D \rightarrow \mathbb{R}$ such that $f=0$ on $\partial D$ and $\int_{D} f d M=0$.

Smooth solutions of the isoperimetric problem which seeks the least perimeter that encloses a given volume are stable hypersurfaces with constant mean curvature. So it is important to determine which hypersurfaces are stable. If $\bar{M}$ is a space form, the case $M$ closed has been solved in [3], concluding that $M$ must be a geodesic sphere, and the case $M$ complete, with $m=2$, has been partially solved by several authors (see e.g. the paper of Ritoré and Ros [21] and references therein).

We ask if somehow we can extend these variational properties to higher codimension submanifolds of a Riemannian manifold $\bar{M}$ of dimension $m+n$. We use a pre-calibration $\Omega$ of rank $m+1$ on the ambient space $\bar{M}$ to define the "enclosed volume" of a variation $\bar{\phi}:[0, \varepsilon] \times D \rightarrow \bar{M}$ as the $\Omega$-volume $V_{D}(t)=\int_{[0, t] \times D} \bar{\phi}^{*} \Omega$. If $\bar{M}$ is of dimension $m+1$ and $\Omega$ is its volume element, we recover the case [3]. We will assume $M$ has calibrated extended tangent space, that is, there exists a smooth global unit normal $v$ such that $H=$ $\|H\| \nu$ and $E M=\mathbb{R} \boldsymbol{v} \oplus T M$ is a $\Omega$-calibrated vector bundle. This is a strong restriction, and corresponds in some cases to be able to extend $M$ to a calibrated $(m+1)$-dimensional submanifold $M^{\prime}$ such that $T M^{\prime}=E M$ along $M$. Even in this case, our approach differs from [3], for we allow $\phi_{t}$ to take values outside $M^{\prime}$, and so our enclosed volume at each time $t$ may not correspond to the enclosed volume in $M^{\prime}$ defined in [3].

Variational characterizations of prescribed mean curvature was the subject of earlier work of Gulliver [15, 16], and Duzaar and Fuchs [8, 9]. In [16] a stationary submanifold of a functional $A(D)+\int_{D} \alpha$, where $\alpha$ is an $m$-form such that $H=(d \alpha)^{\sharp}$, prescribes the mean curvature $H$ of $M$ as an alternating $m$-tensor whose values are orthogonal to each of its $m$ arguments. For a submanifold $M$ of a Euclidean space $\mathbb{R}^{m+n}$, Morgan in [19] defined a prescribed enclosed multi-volume, and proved that $M$ is stationary for area for that prescribed multi-volume if and only if, for some $\xi \in \Lambda_{m+1} \mathbb{R}^{m+n}$, the mean curvature of $M$ satisfies $H=\xi\lfloor\vec{S}$, where $\vec{S}$ is the unit $m$-plane tangent to $M$. This corresponds to our condition of an $\Omega$-calibrated extended tangent space $E M$, if $\xi=$ $\|H\| \Omega$, defining a calibration $\Omega$ (see Lemma 2.3). Existence and regularity of such area-minimizing submanifolds (as rectifiable currents), with given boundary and multivolume, are proved in [19] under quite general conditions.

We show that submanifolds with constant mean curvature are just the critical points of $A_{D}(t)$ for variations that fix the $\Omega$-volume, or equivalently, of $J_{D}(t)$ for any variation fixing the boundary $\partial D$. Furthermore, under certain conditions on $\Omega$, it turns out these submanifolds have parallel mean curvature. We compute the second variation of $J_{D}(t)$ and obtain $J_{D}^{\prime \prime}(0)=\int_{D} \bar{g}\left(\mathscr{J}_{\Omega, D}^{\prime}\left(W^{\perp}\right), W^{\perp}\right) d M=: I_{\Omega}\left(W^{\perp}, W^{\perp}\right)$, where $W=\frac{\partial \bar{\phi}}{\partial t}$ at $t=0$, and

$$
\mathscr{J}_{\Omega, D}^{\prime}(W)=-\Delta^{\perp} W^{\perp}-\bar{R}\left(W^{\perp}\right)-\tilde{B}\left(W^{\perp}\right)+m\|H\| C_{\Omega}\left(W^{\perp}\right)-\Psi_{\Omega, D}\left(W^{\perp}\right) v
$$


is the $\Omega$-Jacobi operator acting on sections $W$ of $T \bar{M}$ along $M$. This is the usual Jacobi operator with an extra first-order differential operator $C_{\Omega}$ depending on $\Omega$ and $\bar{\nabla} \Omega$, and $\Psi_{\Omega, D}$ a suitable linear function. We define a class of vector fields of $\bar{M}$ along $\phi: D \rightarrow \bar{M}$,

$$
\overline{\mathscr{F}}_{D, \Omega}=\left\{W=f(v+N): f \in \mathscr{F}_{D}, N \in C^{\infty}\left(\phi^{-1} T \bar{M}\right), N \perp v\right\},
$$

and $\mathscr{F}_{D, \Omega}=\overline{\mathscr{F}}_{D, \Omega} \cap C^{\infty}\left(N M_{/ D}\right)$. An element $W \in \overline{\mathscr{F}}_{D, \Omega}$ satisfies $W=0$ on $\partial D$ and

$$
\int_{D} \Omega\left(W, d \phi\left(e_{1}\right), \ldots, d \phi\left(e_{m}\right)\right) d M=\int_{D} \bar{g}(W, v) d M=0
$$

where $e_{i}$ is a direct o.n. frame of $M$. Such vector fields are vectors of variation for some $\Omega$-volume preserving variations. We will say that a submanifold with parallel mean curvature is $\Omega$-stable if $I_{\Omega}(W, W) \geq 0$ for all vector variations $W$ lying in $H_{0, T}^{1}\left(N M_{/ D}\right)$, i.e., the $H^{1}$-completion of the vector space generated by $\mathscr{F}_{D, \Omega}$. If a calibrated extension $M^{\prime}$ of $M$ exists, our stability condition is more restrictive than the one in [3], and depends on the geometry of $\bar{M}$. But the two concepts are related, if, for example, $\Omega$ is a parallel calibration and $N M$ is a trivial bundle, or it is defined by a fibration of $\bar{M}$ by totally geodesic $(m+1)$-dimensional submanifolds. Related to this last case, we study the $\Omega$-stability of $m$-dimensional geodesic spheres of $(m+n)$-dimensional space forms. For the case $\bar{M}=\mathbb{R}^{m+n}$ with any parallel calibration, we give some natural conditions in Theorem 4.2, which extend the case $n=1$ of [2] and enable us to conclude that a $m$-dimensional stable closed submanifold must be pseudo-umbilical or even a Euclidean sphere. A first difficulty in the general case $n \geq 2$ arises from the fact that a calibrated submanifold $M^{\prime}$ does not have to be totally geodesic, and stability, with no further assumptions, does not seem to imply this. The Hodge theory of spheres yields other conditions on $\Omega$ that are necessary for their $\Omega$-stability in Euclidean spaces (Proposition 4.5).

\section{Critical area under volume constraints}

We consider $\bar{M}$ with Riemannian metric $\bar{g}$ and a fixed $(m+1)$-form $\Omega$, and $\phi: M \rightarrow \bar{M}$ an immersed oriented submanifold. We use $\nabla, \nabla^{\perp}$ and $\bar{\nabla}$ to denote the connections on $M, N M$ and $\bar{M}$, respectively, and $B$ the second fundamental form of $\phi$, as a tensor with values on the normal bundle $N M$.

Let $D \subset M$ be a compact domain with smooth boundary, and $\bar{\phi}:(-\varepsilon, \varepsilon) \times D \rightarrow \bar{M}$, $\bar{\phi}(t, p)=\phi_{t}(p)$, a smooth variation of $\phi=\phi_{0}: D \rightarrow \bar{M}$ that fixes the boundary $\forall t$. Then the vector variation $W_{t}(p)=\frac{\partial \bar{\phi}}{\partial t}(t, p)$ vanishes at $\partial D$. If $M$ is closed ( that is, compact without boundary) we may consider $D=M$. We denote by $d M_{t}$ the volume element of $M_{t}=\left(M, g_{t}\right)$, where $g_{t}=\phi_{t}^{*} \bar{g}$, and by $B_{t}$ the second fundamental form of $M_{t}$. The mean curvature vector is $H_{t}=\frac{1}{m}$ trace $_{g_{t}} B_{t}=\sum_{i j} \frac{1}{m} g_{t}^{i j}\left(\bar{\nabla}_{e_{i}} e_{j}\right)^{\perp}$, where $e_{i}$ is an oriented $g$-o.n. 
frame $\left(g=g_{0}, M=M_{0}\right.$, etc..), $\left(g_{t}\right)_{i j}=g_{t}\left(e_{i}, e_{j}\right)$, and $\top$ and $\perp$ represent the orthogonal projection of $T \bar{M}$ onto $T M_{t}$ and onto the normal bundle $N M_{t}$ of $M_{t}$, respectively. $M$ has parallel (constant, resp.) mean curvature if $H$ is a parallel section in the normal bundle ( $\|H\|$ is constant, resp.). The area of $D_{t}=\left(D, g_{t}\right)$ and the $\Omega$-volume of $\bar{\phi}$ are respectively given by

$$
A_{D}(t)=\int_{D} d M_{t}, \quad V_{D}(t):=\int_{[0, t] \times D} \bar{\phi}^{*} \Omega .
$$

Lemma 2.1. For a local direct g-o.n. frame $e_{i}$ of $M$,

$$
\begin{aligned}
A_{D}^{\prime}(t) & =-\int_{D} m \bar{g}\left(H_{t}, W_{t}\right) d M_{t} \\
V_{D}^{\prime}(t) & =\int_{D} \Omega\left(W_{t}(p), d \phi_{t}(p)\left(e_{1}\right), \ldots, d \phi_{t}(p)\left(e_{m}\right)\right) d M .
\end{aligned}
$$

In particular, $A_{D}^{\prime}(t)$ and $V_{D}^{\prime}(t)$ depend only on $W_{t}^{\perp} \in N M_{t}$.

Proof. The formula for $A_{D}^{\prime}(t)$ is very well known, but we recall here some formulas that we will need to use in section 3. Set $g_{i j}(t, p)=g_{t}\left(e_{i}, e_{j}\right)$. Then $A_{D}(t)=$ $\int_{D} d M_{t}\left(e_{1}, \ldots, e_{m}\right) d M=\int_{D} \sqrt{\operatorname{det}\left[g_{i j}(t, p)\right]} d M$. Using the Hessian of $\bar{\phi}$, as a map from $(-\varepsilon, \varepsilon) \times D$ with metric $d t^{2}+g$, we see that, at $t=0$ and $p \in D, \bar{\nabla}_{\frac{d}{d t}}\left(d \phi_{t}\left(e_{i}\right)\right)=\bar{\nabla}_{e_{i}} W$. Thus,

$$
\left.\frac{d}{d t}\right|_{t=0} g_{i j}=\bar{g}\left(\bar{\nabla}_{e_{i}} W, d \phi\left(e_{j}\right)\right)+\bar{g}\left(\bar{\nabla}_{e_{j}} W, d \phi\left(e_{i}\right)\right),
$$

and so $\left.\frac{d}{d t}\right|_{t=0}\left(\operatorname{det}\left[g_{i j}(t, p)\right]\right)^{\frac{1}{2}}=\sum_{i} \bar{g}\left(\bar{\nabla}_{e_{i}} W, d \phi\left(e_{i}\right)\right)=\operatorname{div}_{M}\left(W^{\top}\right)-m \bar{g}\left(H, W^{\perp}\right)$. Therefore, $A^{\prime}(0)=-\int_{D} m \bar{g}\left(H, W^{\perp}\right) d M$. The same formula holds for any $t$. Now for any $0 \leq s \leq t$ and $p \in D, \bar{\phi}^{*} \Omega(s, p)=\Omega\left(\frac{\partial \bar{\phi}}{\partial t}(s, p), d \phi_{s}(p)\left(e_{1}\right), \ldots, d \phi_{s}(p)\left(e_{m}\right)\right) d s \wedge d M$. Hence

$$
V_{D}(t)=\int_{0}^{t}\left(\int_{D} \Omega\left(\frac{\partial \bar{\phi}}{\partial t}(s, p), d \phi_{s}(p)\left(e_{1}\right), \ldots, d \phi_{s}(p)\left(e_{m}\right)\right) d M\right) d s
$$

Differentiation with respect to $t$ proves the lemma.

Definition 2.1. A variation $\phi_{t}$ is said $\Omega$-volume preserving if $V_{D}(t)=V(0)=0 \forall t$.

For each $W \in T_{\phi(p)} \bar{M}$ we set

$$
a_{W}(p)=\Omega\left(W_{p}, d \phi\left(e_{1}\right), \ldots, d \phi\left(e_{m}\right)\right)=a_{W^{\perp}}(p) .
$$

In what follows, $\Omega$ is a rank- $(m+1)$ pre-calibration on $\bar{M}$.

This means $\Omega$ is an $(m+1)$-form on $\bar{M}$ such that $\left|\Omega\left(u_{1}, \ldots, u_{m+1}\right)\right| \leq 1$, for any o.n. system $u_{i}$ of $T_{x} \bar{M}$, and equality holds for some system in $T_{x} \bar{M}$, at each $x \in \bar{M}$. In the 
latter case, we will refer to the subspace $\operatorname{span}\left\{u_{i}\right\}$ as $\Omega$-calibrated. An $\Omega$-calibrated submanifold is an $(m+1)$-dimensional submanifold $M^{\prime}$ with calibrated tangent space ([17]). For these submanifolds, $\Omega$ restricted to $M^{\prime}$ is the volume element of $M^{\prime}$. If $\Omega$ is a calibration, that is, $\Omega$ is a closed form, such submanifolds are homologically area minimizing, and in particular minimal stable in $\bar{M}$. Before we give the next definition, we recall the following Lemma 2.1 of [18]:

Lemma 2.2. If $u_{i}$ is an o.n. system with $\Omega\left(u_{1}, \ldots, u_{m+1}\right)=\cos \theta, \theta \in[0, \pi]$, then for any $w \perp u_{i} \forall i$, and any $j,\left|\Omega\left(w, u_{1}, \ldots, \hat{u}_{j}, \ldots, u_{m+1}\right)\right| \leq \sin \theta$.

Definition 2.2. We will say that an oriented m-dimensional immersed submanifold $\phi$ : $M \rightarrow \bar{M}$ has $\Omega$-calibrated extended tangent space on $D$, iffor each $p \in D$, there exist a unit normal vector $v_{p}$ such that $\bar{g}(H, v)=\|H\|$, and for a direct o.n. frame $e_{i}$ of $T_{p} M$,

$$
a_{v}(p)=\Omega\left(v_{p}, d \phi\left(e_{1}\right), \ldots, d \phi\left(e_{m}\right)\right)=1 .
$$

We will refer to $E M_{p}=\mathbb{R} v_{p} \oplus T_{p} M$ as the extended tangent space of $M$ at $p$ in $\bar{M}$, and denote $B^{v}(X, Y)=\bar{g}(B(X, Y), v)$.

The next lemma ensures that, if $v \in N M_{p}$ satisfies $a_{v}(p)=1$, then $v$ is unique. We will always assume that $v$ defines a smooth global section of $N M_{/ D}$.

Lemma 2.3. If $\phi$ has calibrated extended tangent space, then $\forall W \in N M_{p}, u_{i} \in T_{p} M$,

$$
\begin{aligned}
& \Omega\left(W, v, d \phi\left(u_{1}\right), \ldots, d \phi\left(u_{m-1}\right)\right)=0 \\
& \Omega\left(W, d \phi\left(u_{1}\right), \ldots, d \phi\left(u_{m}\right)\right)=\bar{g}\left(W, v_{p}\right) \sqrt{\operatorname{det}\left[g\left(u_{i}, u_{j}\right)\right]}
\end{aligned}
$$

Furthermore, for any $\bar{W} \in T_{\phi(p)} \bar{M}, \quad \bar{g}(H, \bar{W})=\|H\| a_{\bar{W}}$.

Proof. The equalities are immediate consequences of Lemma 2.2. Then it follows $\bar{g}(\bar{W}, H)=\|H\| \bar{g}(\bar{W}, v)=\|H\| a_{\bar{W}}$.

It is clear that if an $(m+1)$-dimensional submanifold $M^{\prime}$ of $\bar{M}$ contains $M$, the mean curvature of $M$ in $M^{\prime}$ is the same as in $\bar{M}$ only if $T M^{\prime}=E M$ along $M$. This is the case when $M^{\prime}$ is totally geodesic in $\bar{M}$. We do not know if a calibrated $(m+1)$-dimensional submanifold $M^{\prime}$ containing $M$ does exist. Harvey and Lawson [17], using methods of Cartan-Kähler theory, proved that, for some calibrations, the boundaries of $\Omega$-calibrated manifolds are exactly the $m$-dimensional submanifolds $\Gamma$ that are maximally $\Omega$-like, that is, at each $x \in \Gamma$, its tangent space is in the span of a calibrated subspace $E_{x}$. Definition 2.2 is a particular case of this condition. A positive answer to this problem for a given $\Omega$ would be equivalent, at each $x \in \bar{M}$, to prove a modified version of Hilbert's seventeenth problem in $\mathbb{R}^{m+n} \equiv T_{x} \bar{M}$, in the terms formulated in [17]. Thus, if this problem turns out to be true for $\Omega$, an $m$-dimensional submanifold $M$ with calibrated extended tangent space only exists if the mean curvature $H$ points in the same direction of the unit normal of $M$ as a submanifold of the extended calibrated manifold $M^{\prime}$. 
Example 2.1. If $\bar{M}=N^{m+1} \times P^{n-1}$, where $N$ and $P$ are Riemannian manifolds, and $\Omega=\operatorname{Vol}_{N}$, the calibrated submanifolds are the slices $N \times h_{0}$ where $h_{0} \in P$. Let $\phi: M \rightarrow \bar{M}$ with components $\phi(p)=(\psi(p), h(p))$. Then $a_{v}(p)=1$ for all $p$ means that $d \phi\left(e_{i}\right)$ and $v$ lie in $T N$. In particular $d h \equiv 0$, that is, $h$ is constant. Consequently $\phi$ lies in a slice $M^{\prime}=N \times h_{0}$. Furthermore, since $M^{\prime}$ is totally geodesic in $\bar{M}$, then $T M^{\prime}=E M$ along $M$.

Example 2.2. Consider $\mathbb{R}^{8}$ with its octonionic structure and $\Omega$ the Cayley calibration, $\Omega(z, u, v, w)=\bar{g}(z, u \times v \times w)$, using the cross product of 3 vectors in $\mathbb{R}^{8}$ (see chapter IV of [17]). Let $\phi: M^{3} \rightarrow \mathbb{R}^{8}$ be any embedded real-analytic 3-dimensional submanifold. Then there exists a unique 4-dimensional real-analytic Cayley submanifold $N$ that contains $M$ ([17], Theorem 4.3 of Chapter IV). $N$ is characterized as the submanifold whose tangent space at $p \in N$ satisfies $T_{p} N=T_{p} M \oplus \mathbb{R} \mu_{p}$, with $\mu_{p}=d \phi\left(e_{1}\right) \times d \phi\left(e_{2}\right) \times d \phi\left(e_{3}\right)$. Thus $a_{\mu}=1$, and so, if $M$ has $\Omega$-calibrated extended tangent space, then $v=\mu$, and $M$ has a calibrated extension $M^{\prime}=N$ such that $T M^{\prime}=E M$. There are many Cayley submanifolds. They can be seen as the class of minimal 4-submanifolds of $\mathbb{C}^{4}$ with equal Kähler angles, which includes the complex and the special Lagrangian submanifolds.

Example 2.3. [15] 16] Consider the imaginary part of the octonionic space $\mathbb{R}^{7}=\operatorname{Im} \mathbb{R}^{8}$, with o.n. basis $\varepsilon_{1}, \ldots, \varepsilon_{7}$ orthogonal to the scalars $\mathbb{R} 1$, endowed with the associative calibration $\Omega(z, u, v)=\bar{g}(z, u \cdot v)$, where $\cdot$ is the Cayley multiplication (see [17], Chap. IV). Let $H: \mathbb{R}^{7} \times \mathbb{R}^{7} \rightarrow \mathbb{R}^{7}$ be the cross product of two octonions, $H(u, v)=\operatorname{Im}(u \cdot v)=$ $u \times v$. If $r \leq 1$ is fixed and $\Gamma=\{(r \cos \theta, r \sin \theta, \phi(\theta), 0,0,0,0): \theta \in[0,2 \pi]\}$ is the graph of a smooth function $\phi$ over a circle $\Gamma_{0}$ of radius $r$ in the $\left\{\varepsilon_{1}, \varepsilon_{2}\right\}$-plane, and if $\Gamma$ lies in a ball of radius 1 , then $\Gamma$ bounds a surface $D$ of prescribed mean curvature $H\left(e_{1}, e_{2}\right)$, where $e_{1}, e_{2}$ is an o.n. frame of $D$ ([16] 3.6). Furthermore, $\|H\|=1$ is constant. In this case $H=v$ in our setting, since $\Omega\left(H, e_{1}, e_{2}\right)=1$. Thus, $D$ is a surface of constant mean curvature in $\mathbb{R}^{7}$ and with $\Omega$-calibrated extended space. Moreover, if $M=D$ is a real analytic 2-dimensional surface of $\mathbb{R}^{7}$, then $E M=T M^{\prime}$, where $M^{\prime}$ is the unique real analytic associative submanifold of $\mathbb{R}^{7}$ which contains $M$ (see Theorem 4.1 of [17]).

Example 2.4. For any immersed submanifold $\phi: M^{m} \rightarrow \bar{M}$, one has $\|B\|^{2} \geq m\|H\|^{2}$, and equality holds if and only if $\phi$ is totally umbilical (a proof can be found in [2] for the case $n=1$ ). This is equivalent to the second fundamental form being an $N M$ valued multiple of the metric, that is, $B(\cdot, \cdot)=H \otimes g$. Totally umbilical submanifolds of space forms are also space forms and are either totally geodesic or $m$-spheres (see [5]). If $M$ is a closed submanifold of $\mathbb{R}^{m+n}$ and with parallel mean curvature, it is sufficient to assume $\|B\|^{2} \leq \frac{m^{2}}{m-1}\|H\|^{2}$ in order to conclude that $M$ is an $m$-dimensional sphere ([7]). A weaker concept is pseudo-umbilicity, that is, when $\bar{g}(B(\cdot, \cdot), H)=\|H\|^{2} g$. Chen and Yano in [6] proved that $M$ is pseudo-umbilical with parallel mean curvature $H=\|H\| v \neq 0$ if and only if $\phi+\|H\|^{-1} v$ is a constant vector $z$ in $\mathbb{R}^{n+m}$. In this case $M$ is immersed into a hypersphere of $\mathbb{R}^{n+m}$ centered at $z$, and $v$ is parallel to the radius 
vector field $\phi-z$. We now suppose $\bar{M}=\mathbb{R}^{m+n}$ with a pre-calibration $\Omega$, and $M$ is a submanifold with nonzero parallel mean curvature $H$ and calibrated extended space. Let $\mathbb{R}^{*}=\mathbb{R} \backslash\left\{-\|H\|^{-1}\right\}$.

Proposition 2.1. If $M$ is pseudo-umbilical, then $\Phi: M \times \mathbb{R}^{*} \rightarrow \mathbb{R}^{m+n}$, given by $\Phi(p, t)=$ $\phi(p)-t v_{p}$, defines an $\Omega$-calibrated extension $M^{\prime}$ of $M$, with second fundamental form satisfying $B^{M^{\prime}}\left(X^{\prime}, v\right)=0$ for all $X^{\prime} \in T_{(p, 0)} M^{\prime}, p \in M$, and $M$ is totally umbilical in $M^{\prime}$. Furthermore, supposing $M$ is closed, then $M$ is totally umbilical in $\bar{M}$ if and only if $M^{\prime}$ is an $(m+1)$-dimensional vector subspace and $M$ is a Euclidean sphere.

Proof. Using the pseudo-umbilicity assumption, we have for $X \in T_{p} M$

$$
d v(p)(X)=\nabla_{X}^{\perp} v+\sum_{i} \bar{g}\left(d v(p)(X), e_{i}\right) e_{i}=-B^{v}\left(X, e_{i}\right) e_{i}=-\|H\| X
$$

The induced metric in $M \times \mathbb{R}^{*}$ is $g_{(p, t)}^{\prime}=(1+t\|H\|)^{2} g_{p}+d t^{2}$, and $\Phi^{*} \Omega$ takes the value 1 along the $g^{\prime}$-o.n. frame $\left\{-\frac{d}{d t}, e(t)_{i}=(1+t\|H\|)^{-1} e_{i}, i=1, \ldots, m\right\}$. Thus, $M^{\prime}$ is a calibrated extension of $M$. The tangent and the normal bundles of $M^{\prime}$ at $(p, t)$ are naturally identified with the corresponding ones at $(p, 0)$. The global section of $T M^{\prime}, \tilde{v}(p, t)=$ $v_{p}$, extends the parallel section $v$ of the normal bundle of $M$, and satisfies $d \tilde{v}(\tilde{v})=$ $d \tilde{v}\left(-\frac{d}{d t}\right)=0$. Thus, the second fundamental form of $M^{\prime}$ satisfies at $(p, t) B^{M^{\prime}}\left(e_{i}, \tilde{v}\right)=0$, and $B^{M^{\prime}}(\tilde{\boldsymbol{v}}, \tilde{\boldsymbol{v}})=\operatorname{proj}_{N M^{\prime}}(d \tilde{\boldsymbol{v}}(\tilde{\boldsymbol{v}}))=0$. Since $B\left(e_{i}, e_{j}\right)=B^{v}\left(e_{i}, e_{j}\right) v+B^{M^{\prime}}\left(e_{i}, e_{j}\right)$, we conclude that $M$ is totally umbilical in $\bar{M}$ if and only if $B^{M^{\prime}}\left(e_{i}, e_{j}\right)=0$, for all $i j$. This holds if and only if $M^{\prime}$ is totally geodesic. In this case, $M$ is an umbilical hypersurface of a Euclidean space, and supposing $M$ is closed, then by a classical result due to E. Cartan (or using [5, 7]), $M$ must be a sphere.

Henceforth we assume $\phi: M \rightarrow \bar{M}$ has calibrated extended tangent space.

We consider the following class of functions, defined in [2, 3], and a class of vector fields

$$
\begin{gathered}
\mathscr{F}_{D}=\left\{f: D \rightarrow \mathbb{R}: f_{/ \partial D}=0, \quad \int_{D} f d M=0\right\} \\
\mathscr{F}_{D, \Omega}=\overline{\mathscr{F}}_{D, \Omega} \cap C^{\infty}\left(N M_{/ D}\right),
\end{gathered}
$$

where $\overline{\mathscr{F}}_{D, \Omega}$ is defined in (1). We consider the orthogonal split of the normal bundle $N M_{/ D}=\mathbb{R} v \oplus F$. For each section $W \in N M$ we denote the corresponding split

$$
W=W^{v}+W^{F}=f v+W^{F}
$$

Definition 2.3. A variation $\phi_{t}$ of $\phi$ is said to be in $\overline{\mathscr{F}}_{D, \Omega}$ (resp. $\left.\mathscr{F}_{D, \Omega}\right)$ if the vector variation at $t=0, W=\left.\frac{\partial \bar{\phi}}{\partial t}\right|_{t=0}$, lies in $\overline{\mathscr{F}}_{D, \Omega}\left(\right.$ resp. $\left.\mathscr{F}_{D, \Omega}\right)$. 
Lemma 2.4. For any $W \in \overline{\mathscr{F}}_{D, \Omega}$ there exists an $\Omega$-volume preserving variation $\phi_{t}$ of $\phi$ that fixes the boundary and has vector variation $W$. Reciprocally, the vector variation $W$ of any $\Omega$-volume preserving variation $\phi_{t}$ satisfies $\int_{D} a_{W} d M=0$ (not necessarily in $\overline{\mathscr{F}} D, \Omega)$.

Proof. We follow the argument of [3]. As in (1), $W=f N^{\prime}$, where $N^{\prime}=v+N$. Let $\rho:(-\varepsilon, \varepsilon) \times M \rightarrow \bar{M}$ be a variation $\rho(\xi, p)$ such that $\rho(0, p)=\phi(p)$, and $\frac{d \rho}{d \xi}(0, p)=N_{p}^{\prime}$. For example, we may take $\rho(\xi, p)=\exp _{\phi(p)}\left(\xi N_{p}^{\prime}\right)$, where $\exp$ is the exponential of $\bar{M}$. We consider, for each $p \in D$, the solution $\xi(t, p)$ of the initial value problem

$$
\left\{\begin{array}{l}
\frac{d \xi}{d t}(t, p)=\frac{a_{W}(p)}{a(\xi(t, p), p)} \\
\xi(0, p)=0
\end{array}\right.
$$

with $a(\xi, p)=\Omega\left(\frac{d \rho}{d \xi}(\xi, p), d \rho_{\xi}(p)\left(e_{1}\right), \ldots, d \rho_{\xi}(p)\left(e_{m}\right)\right)=\sqrt{\operatorname{det}\left[g_{\xi}\left(e_{i}, e_{j}\right)\right]} a_{\frac{\partial \rho}{\partial \xi}}$, where $e_{1}, \ldots, e_{m}$ is any direct $g$-o.n. basis of $T_{p} M$. Note that $a(0, p)=\bar{g}\left(N^{\prime}, v\right)>0$, and so for $t$ sufficiently small $a(\xi(t, p), p)$ does not vanish. Now $\phi_{t}(p)=\rho(\xi(t, p), p)$ satisfies the conditions of the lemma. Reciprocally, if $\phi_{t}$ is $\Omega$-volume preserving, then $V_{D}^{\prime}(0)=0$, which implies $0=\int_{D} \Omega\left(W_{p}, d \phi\left(e_{1}\right), \ldots, d \phi\left(e_{m}\right)\right) d M=\int_{D} a_{W} d M=\int_{D} f d M$.

The first part (a) of the next lemma is due to [2]. If $D=M$ is a closed manifold, we show a similar conclusion for the Sobolev space $H^{1}(D)=\left\{f \in L^{2}(D): \exists \nabla f \in L^{2}(D)\right.$ (in the weak sense) $\}$, with the $H^{1}$-inner product

$$
\left\langle f, f^{\prime}\right\rangle_{H^{1}}=\left\langle f, f^{\prime}\right\rangle_{L^{2}}+\left\langle\nabla f, \nabla f^{\prime}\right\rangle_{L^{2}}=\int_{D} f f^{\prime} d M+\int_{D} \bar{g}\left(\nabla f, \nabla f^{\prime}\right) d M .
$$

The $L^{2}$-completion of $\mathscr{F}_{D}$ is the space $L_{T}^{2}(D)$ of $L^{2}(D)$-functions with zero mean value. The $H^{1}$-completion of $\mathscr{F}_{D}$ is $H_{0, T}^{1}(D)=H_{0}^{1}(D) \cap L_{T}^{2}(D)$ (see [4] and recall that the set of functions $f \in C^{\infty}(\bar{D})$ with $f_{/ \partial D}=0$, and $\mathscr{D}(D)$ of the ones with compact support inside $\stackrel{\circ}{D}$, generate the same spaces $L^{2}(D)$ and $\left.H_{0}^{1}(D)\right)$. If $D=M$ is closed, $H_{0}^{1}(M)=H^{1}(M)$.

Lemma 2.5. (a)[2] If $G \in C^{\infty}(D)$ is $L^{2}$-orthogonal to $\mathscr{F}_{D}$, then $G$ is constant. (b) If $D=M$ is closed and $G \in H^{1}(M)$ is $H^{1}$-orthogonal to $\mathscr{F}_{M}$, then $G$ is constant a.e..

Proof. (b) Let $G_{M}=|M|^{-1} \int_{M} G d M$, where $|M|=\int_{M} d M$. Then, as $M$ is bounded, we have $L^{2}(M) \subset L^{1}(M)$ and $G-G_{M} \in H_{0, T}^{1}(M)$. From $\left\langle G, G-G_{M}\right\rangle_{H^{1}}=0$, we have

$$
\int_{M} G^{2} d M-G_{M} \int_{M} G d M=-\int_{M}\|\nabla G\|^{2} d M \leq 0 .
$$

Thus, $\int_{M} G^{2} d M \leq|M|^{-1}\left(\int_{M} G d M\right)^{2} \leq \int_{M} G^{2} d M$, where we have used Cauchy-Schwarz in the last inequality. Hence $\left|\langle G, 1\rangle_{L^{2}}\right|=|G|_{L^{2}}|1|_{L^{2}}$, which implies $G$ is constant a.e.. 
We consider the set

$$
\mathscr{F}_{D}^{\prime}=\mathscr{F}_{D} \cdot C^{\infty}(D)=\left\{f h: f \in \mathscr{F}_{D}, h \in C^{\infty}(D)\right\}
$$

spanning a vector space $\mathbb{R} \mathscr{F}_{D}^{\prime}$ of the finite sums $\sum_{i} f_{i} h_{i}$, where $f_{i} \in \mathscr{F}_{D}$ and $h_{i} \in C^{\infty}(D)$.

Lemma 2.6. If $G \in L^{2}(D)$ (resp. $G \in H_{0}^{1}(D)$ ) is $L^{2}$-orthogonal (resp. $H^{1}$-orthogonal) to $\mathscr{F}_{D}^{\prime}$, then $G=0$ a.e..

Proof. Since $\mathscr{D}(D)$ is $L^{2}$-dense in $L^{2}(D)$, and $H^{1}$-dense in $H_{0}^{1}(D)$, if we prove that $\mathscr{D}(D) \subset \mathscr{F}_{D}^{\prime}$, then we prove the lemma. Let $\varphi \in \mathscr{D}(D)$. We take $D^{\prime}$ a domain such that $\operatorname{supp} \varphi \subset D^{\prime} \subset \bar{D}^{\prime} \subset D$, and $\phi \in \mathscr{D}\left(D^{\prime}\right), \phi \geq 0$, and such that $\phi=1$ on $\operatorname{supp} \varphi$. Let $\phi_{\varepsilon} \in \mathscr{D}(D)$ not identically zero, $\phi_{\varepsilon} \geq 0$, and with compact support inside a small ball $B_{\varepsilon}$ with $\bar{B}_{\varepsilon} \subset D \backslash \bar{D}^{\prime}$. Then we have a function $f \in \mathscr{D}(D)$ given by $\phi$ on $D^{\prime}$, and by $-c \phi_{\varepsilon}$ on $B_{\varepsilon}$, and zero away from these sets, where $c>0$ is the constant defined by $\int_{D} \phi d M=c \int_{D} \phi_{\varepsilon} D M$. Then $f \in \mathscr{F}_{D}$, and $\varphi=f \varphi \in \mathscr{F}_{D}^{\prime}$.

Let $h_{D}=\frac{1}{|D|} \int_{D}\|H\| d M$ be the mean value of $\|H\|$. For a variation $\phi_{t}$ fixing $\partial D$, define

$$
J_{D}(t)=A_{D}(t)+m h_{D} V_{D}(t)
$$

Then $J_{D}^{\prime}(0)=\int_{D} m\left(-\bar{g}\left(H, W^{\perp}\right)+h_{D} a_{W^{\perp}}\right) d M$.

Theorem 2.1. Consider the following statements:

(1) $\|H\|=h_{D}$, is constant in $D$.

(2) $A_{D}^{\prime}(0)=0$ for all $\Omega$-volume preserving variations on $D$ that fix the boundary $\partial D$.

(3) $J_{D}^{\prime}(0)=0$ for all variations on $D$ fixing the boundary $\partial D$.

$\left(2^{\prime}\right)$ the same as $(2)$, and $\left(3^{\prime}\right)$ the same as $(3)$, but for $\overline{\mathscr{F}}_{D, \Omega}\left(\right.$ or $\left.\mathscr{F}_{D, \Omega}\right)$ variations.

The statements are all equivalent.

Proof. From Lemma 2.3, (1) is equivalent to $\bar{g}(H, W)=h_{D} a_{W}$ for all $W_{p} \in T_{p} \bar{M}$, and $p \in D$. Now we prove $(1) \Rightarrow(2)\left(\Rightarrow\left(2^{\prime}\right)\right)$. For an $\Omega$-volume preserving variation with vector variation $W$, by Lemma $2.1 A_{D}^{\prime}(0)=\int_{D}-m \bar{g}(H, W) d M=-m h_{D} \int_{D} a_{W} d M$. The latter is zero by Lemma 2.4. Next we prove $\left(2^{\prime}\right) \Rightarrow(1)$. If $W \in \overline{\mathscr{F}}_{D, \Omega}$ (or $W \in \mathscr{F}_{D, \Omega}$ ), we we may take $\phi_{t}$ a $\Omega$-volume preserving variation with vector variation $W$ (see Lemma 2.4). Since $\bar{g}(W, H)=\|H\| a_{W}$ (by Lemma 2.3) and by assumption $\int_{D} \bar{g}(H, W) d M=0$, we have $\int_{D}\|H\| a_{W} d M=0$. Considering any function $f \in \mathscr{F}_{D}$ and $W=f v$, we have $a_{W}=f$ and conclude that $\int_{D} f\|H\| d M=0$. Lemma 2.5(a) gives $\|H\|$ constant. Both $(3) \Rightarrow(2),\left(3^{\prime}\right) \Rightarrow\left(2^{\prime}\right)$ and $(1) \Rightarrow(3),(1) \Rightarrow\left(3^{\prime}\right)$ are obvious using Lemmas 2.1 and 2.3 . 


\section{The second variation}

Let $\phi: D \subset M \rightarrow \bar{M}$ be an immersion with constant mean curvature $H$ and with calibrated extended tangent space. In Theorem 2.1 we have shown that $\phi$ is a critical point of $J_{D}(t)$, for all variations fixing the boundary $\partial D$. The Laplacian for sections in the normal bundle is given by $\Delta^{\perp} W^{\perp}=\sum_{i} \nabla_{e_{i}}^{\perp} \nabla_{e_{i}}^{\perp} W^{\perp}-\nabla_{\nabla_{e_{i}} e_{i}} W^{\perp}$. We use the curvature sign of $\bar{M}$, $\bar{R}(X, Y)=-\left[\bar{\nabla}_{X}, \bar{\nabla}_{Y}\right]+\bar{\nabla}_{[X, Y]}$, and set

$$
\begin{aligned}
& \bar{R}\left(W^{\perp}\right)=\sum_{i}\left(\bar{R}\left(d \phi\left(e_{i}\right), W^{\perp}\right) d \phi\left(e_{i}\right)\right)^{\perp}, \\
& \tilde{B}\left(W^{\perp}\right)=\sum_{i j} \bar{g}\left(W^{\perp}, B\left(e_{i}, e_{j}\right)\right) B\left(e_{i}, e_{j}\right) .
\end{aligned}
$$

We also define a differential operator, $C_{\Omega}: C^{\infty}\left(\phi^{-1} T \bar{M}\right) \rightarrow C^{\infty}\left(\phi^{-1} T \bar{M}\right)$, given by

$$
\begin{aligned}
& \int_{D} \bar{g}\left(C_{\Omega}(W), W^{\prime}\right) d M= \\
& =\int_{D}\left(\sum_{i} \frac{1}{2}\left(\Omega\left(W^{\perp}, e_{1}, \ldots, \nabla_{e_{i}}^{\perp} W^{\prime \perp}, \ldots, e_{m}\right)+\Omega\left(W^{\prime \perp}, e_{1}, \ldots, \nabla_{e_{i}}^{\perp} W^{\perp}, \ldots, e_{m}\right)\right)\right. \\
& \left.\quad+\frac{1}{2}\left(\left(\bar{\nabla}_{W^{\perp}} \Omega\right)\left(W^{\perp}, d \phi\left(e_{1}\right), \ldots, d \phi\left(e_{m}\right)\right)+\left(\bar{\nabla}_{W^{\prime}} \Omega\right)\left(W^{\perp}, d \phi\left(e_{1}\right), \ldots, d \phi\left(e_{m}\right)\right)\right)\right) d M .
\end{aligned}
$$

If we use the identity $\bar{\nabla}_{e_{i}} W^{\prime \perp}=\nabla_{e_{i}}^{\perp} W^{\prime \perp}-\sum_{j} \bar{g}\left(B\left(e_{i}, e_{j}\right), W^{\prime \perp}\right) e_{j}$, and define a vector field $X_{W, W^{\prime}}$ by $g\left(X_{W, W^{\prime}}, e_{i}\right)=\Omega\left(W^{\perp}, d \phi\left(e_{1}\right), \ldots, W^{\prime \perp}, \ldots, d \phi\left(e_{m}\right)\right)$, with $W^{\perp \perp}$ in the $i$ position, then applying Lemma 2.3 we may write the first term of this operator as

$$
\begin{aligned}
& \sum_{i} \Omega\left(W^{\perp}, d \phi\left(e_{1}\right), \ldots, \nabla_{e_{i}}^{\perp} W^{\prime \perp}, \ldots, d \phi\left(e_{m}\right)\right)= \\
& =\sum_{i} \Omega\left(W^{\prime \perp}, d \phi\left(e_{1}\right), \ldots, \nabla_{e_{i}}^{\perp} W^{\perp}, \ldots, d \phi\left(e_{m}\right)\right)-\bar{\nabla}_{e_{i}} \Omega\left(W^{\perp}, d \phi\left(e_{1}\right), \ldots, W^{\perp}, \ldots, d \phi\left(e_{m}\right)\right) \\
& \quad-\sum_{i} \sum_{j \neq i} \Omega\left(W^{\perp}, d \phi\left(e_{1}\right), \ldots, B\left(e_{i}, e_{j}\right), \ldots, W^{\prime \perp}, \ldots, d \phi\left(e_{m}\right)\right)+\operatorname{div}\left(X_{W W^{\prime}}\right) .
\end{aligned}
$$

Upon integration, $\operatorname{div}\left(X_{W W^{\prime}}\right)$ vanishes for $W^{\prime}$ with compact support in $D^{\circ}$. Thus, $C_{\Omega}$ is an $L^{2}$-self-adjoint first-order differential operator, only depends on $C^{\infty}\left(N M_{/ D}\right)$, and takes values on $C^{\infty}\left(N M_{/ D}\right)$. If $\nabla^{\perp} v=0$, and denoting by $\nabla^{F}$ the connection on $F$, by Lemma 2.3 we see that $\Omega\left(W^{\perp}, d \phi\left(e_{1}\right), \ldots, \nabla_{e_{i}}^{\perp} W^{\prime \perp}, \ldots, d \phi\left(e_{m}\right)\right)=\Omega\left(W^{F}, d \phi\left(e_{1}\right), \ldots, \nabla_{e_{i}}^{F} W^{\prime F}, \ldots\right.$, $\left.d \phi\left(e_{m}\right)\right)$. In this case, and if moreover $\bar{\nabla} \Omega=0$, then $\bar{g}\left(C_{\Omega}(W), v\right)=0$ holds for all $W$.

Lemma 3.1. For a variation $\phi_{t}$ that fixes the boundary and with vector variation $W$,

$$
J^{\prime \prime}{ }_{D}(0)=\int_{D} \bar{g}\left(-\Delta^{\perp} W^{\perp}-\bar{R}\left(W^{\perp}\right)-\tilde{B}\left(W^{\perp}\right)+m\|H\| C_{\Omega}\left(W^{\perp}\right), W^{\perp}\right) d M .
$$

In particular $J_{D}^{\prime \prime}(0)$ only depends on $W^{\perp}$. 
Proof. Let $e_{i}^{t}$ be a $g_{t}$-o.n. with $e_{i}^{0}=e_{i}$. We have

$$
\begin{aligned}
& a_{W_{t}^{\perp}}=\Omega\left(W_{t}^{\perp}, d \phi_{t}\left(e_{1}^{t}\right), \ldots, d \phi_{t}\left(e_{m}^{t}\right)\right)=\frac{\Omega\left(W_{t}^{\perp}, d \phi_{t}\left(e_{1}\right), \ldots, d \phi_{t}\left(e_{m}\right)\right)}{\left(\sqrt{\left.\operatorname{det}\left[g_{t}\left(e_{i}, e_{j}\right)\right]\right)}\right.}, \\
& J_{D}^{\prime}(t)=\int_{D} m\left(-\bar{g}\left(H_{t}, W_{t}^{\perp}\right)+h_{D} a_{W_{t}^{\perp}}\right) d M_{t}\left(e_{1}, \ldots, e_{m}\right) d M .
\end{aligned}
$$

By Lemma 2.3, at $t=0, \bar{g}\left(H_{t}, W_{t}^{\perp}\right)=h_{D} a_{W_{t}^{\perp}}$. Therefore,

$$
J_{D}^{\prime \prime}(0)=\left.\int_{D} \frac{d}{d t}\right|_{t=0}\left(-m \bar{g}\left(H_{t}, W_{t}^{\perp}\right)+m h_{D} a_{W_{t}^{\perp}}\right) d M .
$$

Now we have

$$
\left.\frac{d}{d t}\right|_{t=0} \bar{g}\left(H_{t}, W_{t}^{\perp}\right)=\bar{g}\left(\bar{\nabla}_{\left.\frac{d}{d t}\right|_{t=0}} H_{t}, W^{\perp}\right)+\bar{g}\left(H, \bar{\nabla}_{\left.\frac{d}{d t}\right|_{t=0}} W_{t}^{\perp}\right) .
$$

The next formula is well known for $W^{\top}=0([22])$, but we prove here the general case

$$
\bar{g}\left(\bar{\nabla}_{\left.\frac{d}{d t}\right|_{t=0}} m H_{t}, W^{\perp}\right)=\bar{g}\left(\Delta^{\perp} W^{\perp}+\bar{R}\left(W^{\perp}\right)+\tilde{B}\left(W^{\perp}\right)+m \nabla_{W^{\top}}^{\perp} H, W^{\perp}\right) .
$$

In (9), if $n=1$, the term $\nabla^{\perp} H$ vanishes, giving the formula in [2, 3]. At a fixed point $p_{0} \in M$ we consider local $g$-o.n. frames $e_{i}$ such that $\nabla e_{i}\left(p_{0}\right)=0$, and set $g_{i j}(t, p)=$ $g_{t}\left(e_{i}, e_{j}\right)$. Since $m H_{t}=\sum_{i j} g_{t}^{i j} B_{t}\left(e_{i}, e_{j}\right)$, then at $t=0$ and $p=p_{0}$

$$
\bar{\nabla}_{\left.\frac{d}{d t}\right|_{t=0}} m H_{t}=\sum_{i j}\left(\left.\frac{d}{d t}\right|_{t=0} g^{i j}\right) B\left(e_{i}, e_{j}\right)+\sum_{i} \bar{\nabla}_{\left.\frac{d}{d t}\right|_{t=0}}\left(B_{t}\left(e_{i}, e_{i}\right)\right) .
$$

Using the symmetry of $B$, eq. (2), and $\left.\frac{d}{d t}\right|_{t=0} g^{i j}=-\left.\frac{d}{d t}\right|_{t=0} g_{i j}$, we have

$$
\sum_{i j}\left(\left.\frac{d}{d t}\right|_{t=0} g^{i j}\right) B\left(e_{i}, e_{j}\right)=\sum_{i}-2 B\left(e_{i}, \nabla_{e_{i}} W^{\top}\right)+\sum_{i j} 2 \bar{g}\left(W^{\perp}, B\left(e_{i}, e_{j}\right)\right) B\left(e_{i}, e_{j}\right) .
$$

On the other hand, at $t=0$ and $p=p_{0}$,

$$
\begin{aligned}
\sum_{i} \bar{\nabla}_{\frac{d}{d t} l_{t=0}}\left(B_{t}\left(e_{i}, e_{i}\right)\right)=\sum_{i} \bar{\nabla}_{\frac{d}{d t}}\left(\left(\bar{\nabla}_{e_{i}}\left(d \phi_{t}\left(e_{i}\right)\right)^{\perp}\right)\right. \\
=\sum_{i} \bar{\nabla}_{\frac{d}{d t}}\left(\bar{\nabla}_{e_{i}}\left(d \phi_{t}\left(e_{i}\right)\right)-\sum_{k u} g_{t}^{k u} \bar{g}\left(\bar{\nabla}_{e_{i}}\left(d \phi_{t}\left(e_{i}\right)\right), d \phi_{t}\left(e_{k}\right)\right) d \phi_{t}\left(e_{u}\right)\right) \\
=\sum_{i} \bar{\nabla}_{\frac{d}{d t}}\left(\bar{\nabla}_{e_{i}}\left(d \phi_{t}\left(e_{i}\right)\right)\right)-\sum_{i k u}\left(\frac{d}{d t} g_{t}^{k u}\right) g\left(\nabla_{e_{i}} e_{i}\left(p_{0}\right), e_{k}\right) d \phi\left(e_{u}\right) \\
\quad-\sum_{i, k} \bar{g}\left(\bar{\nabla}_{\frac{d}{d t}}\left(\bar{\nabla}_{e_{i}}\left(d \phi_{t}\left(e_{i}\right)\right)\right), d \phi\left(e_{k}\right)\right) d \phi\left(e_{k}\right)-\bar{g}\left(\bar{\nabla}_{e_{i}}\left(d \phi\left(e_{i}\right)\right), \bar{\nabla}_{\frac{d}{d t}}\left(d \phi_{t}\left(e_{k}\right)\right)\right) d \phi\left(e_{k}\right) \\
\quad-\sum_{i, k} \bar{g}\left(\bar{\nabla}_{e_{i}}\left(d \phi\left(e_{i}\right)\right), d \phi\left(e_{k}\right)\right) \bar{\nabla}_{\frac{d}{d t}}\left(d \phi_{t}\left(e_{k}\right)\right)
\end{aligned}
$$




$$
\begin{aligned}
& =\sum_{i}\left(\bar{\nabla}_{\frac{d}{d t}}\left(\bar{\nabla}_{e_{i}}\left(d \phi_{t}\left(e_{i}\right)\right)\right)\right)^{\perp}-\sum_{k} \bar{g}\left(m H, \bar{\nabla}_{e_{k}} W\right) d \phi\left(e_{k}\right) \\
& =\sum_{i}\left(\bar{\nabla}_{e_{i}}\left(\bar{\nabla}_{\frac{d}{d t}}\left(d \phi_{t}\left(e_{i}\right)\right)\right)+\bar{R}\left(d \phi\left(e_{i}\right), W\right) d \phi\left(e_{i}\right)\right)^{\perp}-\sum_{k} \bar{g}\left(m H, \bar{\nabla}_{e_{k}} W\right) d \phi\left(e_{k}\right) \\
& =\left(\sum_{i} \bar{\nabla}_{e_{i}} \bar{\nabla}_{e_{i}} W+\bar{R}\left(d \phi\left(e_{i}\right), W\right) d \phi\left(e_{i}\right)\right)^{\perp}-\sum_{k} \bar{g}\left(m H, \bar{\nabla}_{e_{k}} W\right) d \phi\left(e_{k}\right) .
\end{aligned}
$$

We note that

$$
\begin{aligned}
\left(\bar{\nabla}_{e_{i}} \bar{\nabla}_{e_{i}} W^{\perp}\right)^{\perp} & =\left(\bar{\nabla}_{e_{i}}\left(\sum_{j} \bar{g}\left(\bar{\nabla}_{e_{i}} W^{\perp}, d \phi\left(e_{j}\right)\right) d \phi\left(e_{j}\right)+\nabla_{e_{i}}^{\perp} W^{\perp}\right)\right)^{\perp} \\
& =\left(\sum_{j} \bar{\nabla}_{e_{i}}\left(-\bar{g}\left(W^{\perp}, B\left(e_{i}, e_{j}\right)\right) d \phi\left(e_{j}\right)\right)\right)^{\perp}+\nabla_{e_{i}}^{\perp} \nabla_{e_{i}}^{\perp} W^{\perp} \\
& =\sum_{j}-\bar{g}\left(W^{\perp}, B\left(e_{i}, e_{j}\right)\right) B\left(e_{i}, e_{j}\right)+\nabla_{e_{i}}^{\perp} \nabla_{e_{i}}^{\perp} W^{\perp}, \\
\left(\bar{\nabla}_{e_{i}} \bar{\nabla}_{e_{i}} W^{\top}\right)^{\perp} & =\left(\bar{\nabla}_{e_{i}}\left(\nabla_{e_{i}} W^{\top}+B\left(e_{i}, W^{\top}\right)\right)\right)^{\perp} \\
& =\left(\bar{\nabla}_{e_{i}}\left(\nabla_{e_{i}} W^{\top}\right)\right)^{\perp}+\nabla_{e_{i}} B\left(e_{i}, W^{\top}\right)+B\left(e_{i}, \nabla_{e_{i}} W^{\top}\right) \\
& =2 B\left(e_{i}, \nabla_{e_{i}} W^{\top}\right)+\nabla_{W^{\top}} B\left(e_{i}, e_{i}\right)-\left(\bar{R}\left(d \phi\left(e_{i}\right), W^{\top}\right) d \phi\left(e_{i}\right)\right)^{\perp},
\end{aligned}
$$

where in the last equality we have used Coddazzi's equation. Here $\nabla B$ denotes the covariant derivative of $B$ as a tensor with values in $N M$. Therefore,

$$
\bar{\nabla}_{\left.\frac{d}{d t}\right|_{t=0}} m H_{t}=\Delta^{\perp} W^{\perp}+\tilde{B}\left(W^{\perp}\right)+\bar{R}\left(W^{\perp}\right)+\nabla_{W^{\top}}^{\perp} m H-\sum_{k} \bar{g}\left(m H, \bar{\nabla}_{e_{k}} W\right) d \phi\left(e_{k}\right)
$$

and we obtain (9). Next we calculate $\left.\frac{d}{d t}\right|_{t=0} a_{W_{t}^{\perp}}$.

$$
\begin{aligned}
& \left.\frac{d}{d t}\right|_{t=0} a_{W_{t}^{\perp}}=\left.\frac{d}{d t}\right|_{t=0} \Omega\left(W_{t}^{\perp}, d \phi_{t}\left(e_{1}\right), \ldots, d \phi_{t}\left(e_{m}\right)\right)+\left.\bar{g}\left(W^{\perp}, v\right) \frac{d}{d t}\right|_{t=0}\left(\operatorname{det}\left[g_{t}\left(e_{i}, e_{j}\right)\right]\right)^{-\frac{1}{2}} \\
& =\left(\bar{\nabla}_{\left.\frac{d \phi_{t}}{d t}\right|_{t=0}} \Omega\right)\left(W^{\perp}, d \phi\left(e_{1}\right), \ldots, d \phi\left(e_{m}\right)\right)+\Omega\left(\bar{\nabla}_{\left.\frac{d}{d t}\right|_{t=0}} W_{t}^{\perp}, d \phi\left(e_{1}\right), \ldots, d \phi\left(e_{m}\right)\right) \\
& +\sum_{i} \Omega\left(W^{\perp}, d \phi\left(e_{1}\right), \ldots, \bar{\nabla}_{\left.\frac{d}{d t}\right|_{t=0}}\left(d \phi_{t}\left(e_{i}\right)\right), \ldots, d \phi\left(e_{m}\right)\right)-\bar{g}\left(W^{\perp}, v\right) \bar{g}\left(\bar{\nabla}_{e_{i}} W, d \phi\left(e_{i}\right)\right) .
\end{aligned}
$$

Applying Lemma 2.3, and $\bar{\nabla}_{\left.\frac{d}{d t}\right|_{t=0}}\left(d \phi_{t}\left(e_{i}\right)\right)=\bar{\nabla}_{e_{i}} W=\left(\bar{\nabla}_{e_{i}} W\right)^{\top}+\nabla_{e_{i}}^{\perp} W^{\perp}+B\left(e_{i}, W^{\top}\right)$, we have

$$
\begin{aligned}
& \Omega\left(W^{\perp}, d \phi\left(e_{1}\right), \ldots, \bar{\nabla}_{\left.\frac{d}{d t}\right|_{t=0}}\left(d \phi_{t}\left(e_{i}\right)\right), \ldots, d \phi\left(e_{m}\right)\right)=\bar{g}\left(W^{\perp}, v\right) \bar{g}\left(\bar{\nabla}_{e_{i}} W, d \phi\left(e_{i}\right)\right)+ \\
& +\sum_{i} \Omega\left(W^{\perp}, d \phi\left(e_{1}\right), \ldots, \nabla_{e_{i}}^{\perp} W^{\perp}, \ldots, d \phi\left(e_{m}\right)\right)+\Omega\left(W^{\perp}, d \phi\left(e_{1}\right), \ldots, B\left(e_{i}, W^{\top}\right), \ldots, d \phi\left(e_{m}\right)\right) \text {. }
\end{aligned}
$$


Thus,

$$
\begin{aligned}
\left.\frac{d}{d t}\right|_{t=0} a_{W_{t}^{\perp}}= & \left(\bar{\nabla}_{W^{\top}} \Omega\right)\left(W^{\perp}, d \phi\left(e_{1}\right), \ldots, d \phi\left(e_{m}\right)\right)+\bar{g}\left(C_{\Omega}\left(W^{\perp}\right), W^{\perp}\right) \\
& +\bar{g}\left(\bar{\nabla}_{\left.\frac{d}{d t}\right|_{t=0}} W_{t}^{\perp}, v\right)+\sum_{i} \Omega\left(W^{\perp}, d \phi\left(e_{1}\right), \ldots, B\left(e_{i}, W^{\top}\right), \ldots, d \phi\left(e_{m}\right)\right) .
\end{aligned}
$$

Now we observe that, for $X \in T_{p_{0}} M$,

$$
\begin{aligned}
& \left(\bar{\nabla}_{X} \Omega\right)\left(W^{\perp}, d \phi\left(e_{1}\right), \ldots, d \phi\left(e_{m}\right)\right)=d\left(\bar{g}\left(W^{\perp}, v\right)\right)(X)-\Omega\left(\bar{\nabla}_{X} W^{\perp}, d \phi\left(e_{1}\right), \ldots, d \phi\left(e_{m}\right)\right) \\
& \quad-\sum_{i} \Omega\left(W^{\perp}, d \phi\left(e_{1}\right), \ldots, \bar{\nabla}_{X}\left(d \phi\left(e_{i}\right)\right), \ldots, d \phi\left(e_{m}\right)\right) \\
& =d\left(\bar{g}\left(W^{\perp}, v\right)\right)(X)-\bar{g}\left(\bar{\nabla}_{X} W^{\perp}, v\right)-\sum_{i} \Omega\left(W^{\perp}, d \phi\left(e_{1}\right), \ldots, B\left(X, e_{i}\right), \ldots, d \phi\left(e_{m}\right)\right) .
\end{aligned}
$$

Consequently,

$$
\begin{aligned}
& \left(\bar{\nabla}_{X} \Omega\right)\left(W^{\perp}, d \phi\left(e_{1}\right), \ldots, d \phi\left(e_{m}\right)\right)= \\
& \quad=\bar{g}\left(W^{\perp}, \nabla_{X}^{\perp} v\right)-\sum_{i} \Omega\left(W^{\perp}, d \phi\left(e_{1}\right), \ldots, B\left(X, e_{i}\right), \ldots, d \phi\left(e_{m}\right)\right) .
\end{aligned}
$$

Therefore, taking $X=W^{\top}$ in (12), using (11) and (9), and adding (8),

$\left.\frac{d}{d t}\right|_{t=0}\left(m g\left(H_{t}, W_{t}^{\perp}\right)-m\|H\| a_{W_{t}^{\perp}}\right)=\bar{g}\left(\Delta^{\perp} W^{\perp}+\bar{R}\left(W^{\perp}\right)+\tilde{B}\left(W^{\perp}\right)-m\|H\| C_{\Omega}\left(W^{\perp}\right), W^{\perp}\right)$.

From (12) in the preceding proof, we conclude:

Proposition 3.1. If $\phi: M \rightarrow \bar{M}$ is an immersion with calibrated extended tangent space, then $v$ is a parallel section of the normal bundle if and only if, $\forall X \in T_{p} M, W^{\perp} \in N M_{p}$,

$$
\left(\bar{\nabla}_{X} \Omega\right)\left(W^{\perp}, d \phi\left(e_{1}\right), \ldots, d \phi\left(e_{m}\right)\right)=-\sum_{i} \Omega\left(W^{\perp}, d \phi\left(e_{1}\right), \ldots, B\left(X, e_{i}\right), \ldots, d \phi\left(e_{m}\right)\right) .
$$

In this case, if $\phi$ has constant mean curvature, then it has parallel mean curvature.

We now define a self-adjoint strongly elliptic second order differential operator $\mathscr{J}_{\Omega}$ : $C^{\infty}\left(\phi^{-1} T \bar{M}\right) \rightarrow C^{\infty}\left(\phi^{-1} T \bar{M}\right)$,

$$
\mathscr{J}_{\Omega}(W)=\mathscr{J}(W)+m\|H\| C_{\Omega}(W) \quad \in C^{\infty}\left(N M_{/ D}\right),
$$

where $\mathscr{J}(W)$ is the usual Jacobi operator, $\mathscr{J}(W)=-\Delta^{\perp} W^{\perp}-\bar{R}\left(W^{\perp}\right)-\tilde{B}\left(W^{\perp}\right)$. 
We now recall some properties of calibrations defined by fibrations. Consider $\pi$ : $\bar{M} \rightarrow N$ a Riemannian submersion between Riemannian manifolds, defining an orthogonal split of $T \bar{M}$ into the vertical and the horizontal spaces, $T \bar{M}=T \bar{M}^{v} \oplus T \bar{M}^{h}$. For $y \in N, M_{y}^{\prime}=\pi^{-1}(y)$ is the fibre at $y$, which we assume to be of dimension $m+1$, and for $x \in M_{y}^{\prime}, T_{x} \bar{M}^{v}=T_{x}\left(M_{y}^{\prime}\right), T_{x} \bar{M}^{h}=\left(N M_{y}^{\prime}\right)_{x}$. For each vector $X \in T \bar{M}$, we denote by $X^{v}$ and $X^{h}$ its projection into $T \bar{M}^{v}$ and $T \bar{M}^{h}$, respectively. This fibration defines a pre-calibration on $\bar{M}$ that calibrates the fibres $M_{y}^{\prime}$. It is given by

$$
\Omega_{\pi}\left(X_{1}, \ldots, X_{m+1}\right)=\operatorname{Vol}_{y}\left(X_{1}^{v}, \ldots, X_{m+1}^{v}\right), \quad \forall X_{i} \in T_{x} \bar{M}
$$

where $\operatorname{Vol}_{y}$ is the volume element of the fibre $M_{y}^{\prime}$, with $y=\pi(x)$. Let $e_{i}^{\prime}, i=1, \ldots, m+1$ and $e_{\alpha}^{\prime}, \alpha=m+2, \ldots, m+n$ be local o.n. frames of $T \bar{M}^{v}$ and $T \bar{M}^{h}$, respectively.

Lemma 3.2. [18] All components of $\bar{\nabla} \Omega_{\pi}$ and of $d \Omega_{\pi}$ vanish except for the following, where $i, j \leq m+1, \alpha, \beta \geq m+2$ :

$$
\begin{aligned}
& \bar{\nabla}_{e_{j}^{\prime}} \Omega_{\pi}\left(e_{\alpha}^{\prime}, e_{1}^{\prime}, \ldots, \hat{e}_{i}^{\prime}, \ldots, e_{m+1}^{\prime}\right)=(-1)^{i+1} \bar{g}\left(B^{v}\left(e_{j}^{\prime}, e_{i}^{\prime}\right), e_{\alpha}^{\prime}\right) \\
& \bar{\nabla}_{e_{\beta}^{\prime}} \Omega_{\pi}\left(e_{\alpha}^{\prime}, e_{1}^{\prime}, \ldots, \hat{e}_{i}^{\prime}, \ldots, e_{m+1}^{\prime}\right)=(-1)^{i} \bar{g}\left(\bar{\nabla}_{e_{\beta}^{\prime}} e_{\alpha}^{\prime}, e_{i}^{\prime}\right) \\
& d \Omega_{\pi}\left(e_{\alpha}^{\prime}, e_{1}^{\prime}, \ldots, e_{m+1}^{\prime}\right)=-(m+1) \bar{g}\left(H^{v}, e_{\alpha}^{\prime}\right) \\
& d \Omega_{\pi}\left(e_{\alpha}^{\prime}, e_{\beta}^{\prime}, e_{1}^{\prime}, \ldots, \hat{e}_{i}^{\prime}, \ldots, e_{m+1}^{\prime}\right)=(-1)^{i} \bar{g}\left(\left[e_{\alpha}^{\prime}, e_{\beta}^{\prime}\right], e_{i}^{\prime}\right)
\end{aligned}
$$

where $B^{v}$ and $H^{v}$ denote the second fundamental form and the mean curvature of the fibres, respectively.

Proposition 3.2. Assume $M^{\prime}$ is a totally geodesic fibre of a Riemannian submersion $\pi: \bar{M} \rightarrow N$. Furthermore, assume $\phi: M \rightarrow \bar{M}$ is an immersion with $\Omega_{\pi \text {-calibrated }}$ extended tangent space and that $\phi(M)$ lies in $M^{\prime}$ with $E M=T M^{\prime}$ along $M$. Then $v$ is a parallel section of $N M$ and $C_{\Omega}=0$. In particular, if $\phi$ has constant mean curvature, then it has parallel mean curvature.

Proof. We take frames $e_{a}^{\prime}$ such that, at $p \in M, e_{1}^{\prime}=v, e_{i+1}^{\prime}=d \phi\left(e_{i}\right)$, for $i=1, \ldots, m$. The first equality of Lemma 3.2 and $B^{v}=0$ give us $\bar{\nabla}_{e_{i}} \Omega_{\pi}\left(e_{\alpha}^{\prime}, d \phi\left(e_{1}\right), \ldots, d \phi\left(e_{m}\right)\right)=$ 0 . By the Lemma, the component $\bar{\nabla}_{e_{i}} \Omega_{\pi}\left(v, d \phi\left(e_{1}\right), \ldots, d \phi\left(e_{m}\right)\right)$ also vanishes. It is clear that $\Omega_{\pi}\left(W^{\perp}, W^{\perp \perp}, d \phi\left(e_{1}\right), \ldots, d \phi\left(\hat{e}_{i}\right), \ldots, d \phi\left(e_{m}\right)\right)=0$. Applying Proposition 3.1, we conclude $v$ is parallel in $N M$. To prove that $C_{\Omega}=0$ we use the second equality of Lemma 3.2 and the Escobales-O'Neill identity $\left(\bar{\nabla}_{e_{\alpha}^{\prime}} e_{\beta}^{\prime}\right)^{v}=\frac{1}{2}\left[e_{\alpha}^{\prime}, e_{\beta}^{\prime}\right]^{v}$, and that $\bar{\nabla}_{v} \Omega_{\pi}\left(e_{\alpha}^{\prime}, d \phi\left(e_{1}\right), \ldots, d \phi\left(e_{m}\right)\right)=\bar{\nabla}_{e_{a}^{\prime}} \Omega_{\pi}\left(v, d \phi\left(e_{1}\right), \ldots, d \phi\left(e_{m}\right)\right)=0$, for any $a=$ $1, \ldots, m+n$. 


\section{$4 \Omega$-stable submanifolds with parallel mean curvature}

Let $\phi: M \rightarrow \bar{M}$ be an immersed submanifold with calibrated extended tangent space and parallel mean curvature. Given a section $W \in \overline{\mathscr{F}}_{D, \Omega}$, by Lemma 2.4 there is an $\Omega$-volume preserving variation of $\phi$ with vector variation $W$. For such a variation we have $A_{D}^{\prime}(0)=J_{D}^{\prime}(0)=0$ and $A_{D}^{\prime \prime}(0)=J_{D}^{\prime \prime}(0)=\int_{D} \bar{g}\left(\mathscr{J}_{\Omega}\left(W^{\perp}\right), W^{\perp}\right) d M$. We define a symmetric bilinear operator on the vector space $\mathbb{R} \mathscr{\mathscr { F }}_{D, \Omega}$ spanned by $\overline{\mathscr{F}}_{D, \Omega}$

$$
I_{\Omega}\left(W, W^{\prime}\right):=\int_{D} \bar{g}\left(\mathscr{J}_{\Omega}(W), W^{\prime}\right) d M=I_{\Omega}\left(W^{\perp}, W^{\prime \perp}\right) .
$$

We consider the orthogonal split $N M=\mathbb{R} v \oplus F$ into two parallel subbundles. For $f \in$ $\mathscr{F}_{D}$ and $W^{F} \in C^{\infty}(F)$, we have $f v, f\left(v+W^{F}\right) \in \mathscr{F}_{D, \Omega}$. Then $f W^{F} \in \mathbb{R} \mathscr{F}_{D, \Omega}$. Hence, $\mathbb{R} \mathscr{F}_{D, \Omega}=\mathscr{F}_{D} \oplus \mathscr{F}^{\prime}(F)$, where $f \in \mathscr{F}_{D}$ is identified with $f v$, and

$$
\mathscr{F}^{\prime}(F)=\left\{\sum_{a} f_{a} W_{a}^{F}(\text { finite sum }): f_{a} \in \mathscr{F}_{D}, W_{a}^{F} \in C^{\infty}(F)\right\} .
$$

Let $L^{2}\left(N M_{/ D}\right)$ be the space of measurable sections $W$ of the normal bundle such that $\|W\| \in L^{2}(D)$, and $H^{1}\left(N M_{/ D}\right)$ the space of sections $W \in L^{2}\left(N M_{/ D}\right)$ such that $\exists \nabla_{\bar{X}} W \in$ $L^{2}\left(N M_{/ D}\right)$ (in the weak sense) for all $X \in C^{\infty}\left(T M_{/ D}\right)$. We define $L_{T}^{2}\left(N M_{/ D}\right)$ as the $L^{2}$-completion of $\mathbb{R} \mathscr{F}_{D, \Omega}$ in $L^{2}\left(N M_{/ D}\right)$, and $L^{\prime 2}(F)$ the $L^{2}$-completion of $\mathscr{F}^{\prime}(F)$. Then $L_{T}^{2}\left(N M_{/ D}\right)=L_{T}^{2}(D) \oplus L^{\prime 2}(F)$. We also denote by $H_{0}^{\prime 1}(D), H_{0, T}^{1}\left(N M_{/ D}\right), H_{0}^{\prime 1}(F)$ the corresponding $H^{1}$-completion of $\mathbb{R} \mathscr{F}_{D}^{\prime}, \mathbb{R} \mathscr{F}_{D, \Omega}$, and $\mathscr{F}^{\prime}(F)$, respectively, where

$$
\left\langle W, W^{\prime}\right\rangle_{H^{1}}=\int_{D} \bar{g}\left(W, W^{\prime}\right) d M+\int_{M} \sum_{i} \bar{g}\left(\nabla_{e_{i}}^{\perp} W, \nabla_{e_{i}}^{\perp} W^{\prime}\right) d M .
$$

If $D=M$ closed, $H_{0}^{1}=H^{1}$. We consider the quadratic form defined for $W \in \mathbb{R} \mathscr{F}_{D, \Omega}$

$$
Q_{\Omega}(W)=\int_{D}\left(\left\|\nabla^{\perp} W\right\|^{2}-\bar{g}(\bar{R}(W), W)-\bar{g}(\tilde{B}(W), W)+m\|H\| \bar{g}\left(C_{\Omega}(W), W\right)\right) d M .
$$

Then $I_{\Omega}(W, W)=Q_{\Omega}(W)$, and so $I_{\Omega}$ has a natural extension to $W \in H_{0, T}^{1}\left(N M_{/ D}\right)$.

Lemma 4.1. If $Z \in L^{2}\left(N M_{/ D}\right)$ satisfies $\int_{D} \bar{g}\left(Z, W^{\prime}\right) d M=0$ for all $W^{\prime} \in \mathbb{R} \mathscr{F} D, \Omega$, then $Z=c v$ a.e., where $c$ is a constant.

Proof. If we take $W^{\prime}=f v$ where $f \in \mathscr{F}_{D}$, then we conclude by Lemma 2.5(a) that $\bar{g}(Z, v)=c$ a.e. where $c$ is constant. We also have $\int_{D} f \bar{g}\left(Z, W^{F}\right) d M=0$ for all $W^{F} \in$ $C^{\infty}(F)$ and $f \in \mathscr{F}_{D}$. Thus $\bar{g}\left(Z, W^{F}\right)$ is constant a.e. Taking a non-constant function $\rho$ we conclude $\bar{g}\left(Z, \rho W^{F}\right)$ is also constant a.e.. This implies $Z^{F}=0$ a.e.. 
Lemma 4.2. $C_{0}^{\infty}(F)=L^{\prime 2}(F) \cap C_{0}^{\infty}(F)$. In particular $\mathscr{F}^{\prime}(F)$ is $L^{2}$-dense in $C_{0}^{\infty}(F)$.

Proof. First we claim that if $Z \in L^{2}(F)$ and $Z \perp \mathscr{F}^{\prime}(F)$ then $Z=0$ a.e.. To see this, we fix $W \in L^{2}(F)$. Then, for any $f \in \mathscr{F}_{D}, 0=\int_{D} \bar{g}(Z, f W) d M$, which implies by Lemma 2.5(a) that $\bar{g}(Z, W)$ is constant a.e.. Since $W$ is arbitrary, $Z=0$ a.e..Therefore, $L^{\prime 2}(F)=L^{2}(F)$. On the other hand, the $L^{2}$-closure of $C_{0}^{\infty}(F)$ is $L^{2}(F)$.

Lemma 4.3. $\mathbb{R} \mathscr{F}_{D}^{\prime}$ is $H^{1}$-dense in $H_{0}^{1}(D)$, that is, $H_{0}^{\prime 1}(D)=H_{0}^{1}(D)$.

Proof. This is an immediate consequence of Lemma 2.6.

Proposition 4.1. $\mathscr{F}^{\prime}(F)$ is $H^{1}$-dense in $H_{0}^{1}(F)$, that is, $H_{0}^{\prime 1}(F)=H_{0}^{1}(F)$. Furthermore, $H_{0, T}^{1}\left(N M_{/ D}\right)=H_{0, T}^{1}(D) \oplus H_{0}^{1}(F)$ and, for $W \in H_{0}^{1}\left(N M_{/ D}\right)$, W lies in $H_{0, T}^{1}\left(N M_{/ D}\right)$ if and only if $\int_{D} a_{W} d M=0$.

Proof. We only need to prove that any $W \in C_{0}^{\infty}(F)$ with compact support $K \subset \check{D}^{\circ}$ is an element of $\mathscr{F}^{\prime}(F)$, since the set of such sections is $H^{1}$-dense in $H_{0}^{1}(F)$ (see [23]). Let $\varphi \in \mathscr{D}(D)$ with $\varphi=1$ on $K$. We have proved in the proof of Lemma 2.6 that $\varphi \in \mathscr{F}_{D}^{\prime}$, say $\varphi=f h$, as in (4). Then $W=\varphi W=f(h W) \in \mathscr{F}^{\prime}(F)$. The rest is elementary.

Remark 4.1. Assume $M$ is closed and consider $D=M$. In Lemma 4.3 we have shown that the $H^{1}$-closure of $\mathbb{R} \mathscr{F}_{M}^{\prime}$ is $H^{1}(M)$. Let $\phi_{i}, i=0,1 \ldots$, be an $L^{2}$-orthonormal basis of $L^{2}(M)$ of eigenfunctions of $-\Delta$, with corresponding eigenvalues $\lambda_{i} \nearrow+\infty$, where $\lambda_{0}=0$ and $\lambda_{1}>0$. Then $\left\langle\phi_{i}, \phi_{j}\right\rangle_{L^{2}}=\delta_{i j}$ and $\left\langle\nabla \phi_{i}, \nabla \phi_{j}\right\rangle_{L^{2}}=\lambda_{i} \delta_{i j}$. Using integration by parts, i.e., $\int_{M}\|\nabla f\|^{2}=-\int_{M} f \Delta f d M$, we obtain for all $i, j$,

$$
\int_{M}\left\|\nabla\left(\phi_{i} \phi_{j}\right)\right\|^{2} d M=\left(\lambda_{i}+\lambda_{j}\right) \int_{M} \phi_{i}^{2} \phi_{j}^{2} d M-2 \int_{M} \phi_{i} \phi_{j} g\left(\nabla \phi_{i}, \nabla \phi_{j}\right) d M .
$$

On the other hand, $\left\|\nabla\left(\phi_{i} \phi_{j}\right)\right\|^{2}=\phi_{i}^{2}\left\|\nabla \phi_{j}\right\|^{2}+\phi_{j}^{2}\left\|\nabla \phi_{i}\right\|^{2}+2 \phi_{i} \phi_{j} g\left(\nabla \phi_{i}, \nabla \phi_{j}\right)$. Hence,

$$
\int_{M}\left\|\nabla\left(\phi_{i} \phi_{j}\right)\right\|^{2} d M=\frac{\left(\lambda_{i}+\lambda_{j}\right)}{2} \int_{M} \phi_{i}^{2} \phi_{j}^{2} d M+\frac{1}{2} \int_{M}\left(\phi_{i}^{2}\left\|\nabla \phi_{j}\right\|^{2}+\phi_{j}^{2}\left\|\nabla \phi_{i}\right\|^{2}\right) d M .
$$

Consequently,

$$
\int_{M}\left\|\nabla\left(\phi_{i} \phi_{j}\right)\right\|^{2} d M \geq \frac{\left(\lambda_{i}+\lambda_{j}\right)}{2} \int_{M}\left(\phi_{i} \phi_{j}\right)^{2} d M, \quad \int_{M}\left\|\nabla \phi_{i}^{2}\right\|^{2} d M=\frac{4}{3} \lambda_{i} \int_{M} \phi_{i}^{4} d M .
$$

If we take $i \geq 1$ and $j \geq 0, \phi_{i} \phi_{j} \in \mathscr{F}_{M}^{\prime}$ satisfy the above inequalities. But the constant function $h=1 \in H^{1}(M)$ can be expressed as an $L^{2}$-limit of series in terms of $\phi_{i} \phi_{j}$, and it does not satisfy an inequality $\int_{M}\|\nabla h\|^{2} d M \geq c \int_{M} h^{2} d M$, where $c$ is a positive constant. We note that $\phi_{i} \phi_{j}$, with $i \leq j$, is not an orthonormal system. 
Definition 4.1. We will say an immersed submanifold $\phi: M \rightarrow \bar{M}$ of calibrated extended tangent space and of parallel mean curvature is essentially $\Omega$-stable on $D$ if $A^{\prime \prime}{ }_{D}(0) \geq 0$ for all $\Omega$-volume preserving $\overline{\mathscr{F}}_{D, \Omega}$-variations. Equivalently, $\phi$ is essentially $\Omega$-stable on $D$ if and only if $J_{D}^{\prime \prime}(0) \geq 0$ for any variation $\phi_{t}$ with vector variation $W \in \overline{\mathscr{F}}_{D, \Omega}$. We will say $\phi$ is $\Omega$-stable on $D$, if $\forall W \in H_{0, T}^{1}\left(N M_{/ D}\right), I_{\Omega}(W, W) \geq 0$, and $\Omega$-unstable if otherwise.

The equivalence of $A^{\prime \prime}{ }_{D}(0) \geq 0$ with the condition $J_{D}^{\prime \prime}(0) \geq 0$ comes from the fact that $J_{D}^{\prime \prime}(0)$ does not depend on the variation $\bar{\phi}$ but only on the normal component $W^{\perp}$ of the vector variation. The variation $\bar{\phi}$ does not need to be $\Omega$-volume preserving, but one of the variations with vector variation $W \in \overline{\mathscr{F}}_{D, \Omega}$ is $\Omega$-volume preserving.

We consider the linear function on $\mathbb{R} \mathscr{F}_{D, \Omega}, \Psi_{\Omega, D}(W)=|D|^{-1} \int_{D} \bar{g}\left(\mathscr{J}_{\Omega}(W), v\right) d M$, and define a self-adjoint operator, the $\Omega$-Jacobi operator, $\mathscr{J}_{\Omega, D}^{\prime}: \mathbb{R}_{\mathscr{F}_{D, \Omega}} \subset H_{0, T}^{1}\left(N M_{/ D}\right) \rightarrow$ $L_{T}^{2}\left(N M_{/ D}\right)$, given by

$$
\mathscr{J}_{\Omega, D}^{\prime}(W)=\mathscr{J}_{\Omega}(W)-\Psi_{\Omega, D}(W) v .
$$

Then for all $W, W^{\prime} \in \mathbb{R} \mathscr{F}_{D, \Omega}, I_{\Omega}\left(W, W^{\prime}\right)=\int_{D} \bar{g}\left(\mathscr{J}_{\Omega, D}^{\prime}(W), W^{\prime}\right) d M$. We extend the definition of Jacobi field given in [3]:

Definition 4.2. We will say that $W \in H_{0, T}^{1}\left(N M_{/ D}\right) \cap C^{\infty}\left(N M_{/ D}\right)$ is an $\Omega$-Jacobi field along $\phi: D \rightarrow \bar{M}$ if $I_{\Omega}\left(W, W^{\prime}\right)=0, \forall W^{\prime} \in \mathbb{R} \mathscr{F}_{D, \Omega}$.

The next proposition follows immediately from the previous lemmas of this section:

Proposition 4.2. $W$ is an $\Omega$-Jacobi field if and only if $\mathscr{J}_{\Omega}(W)=c v$, where $c$ is a constant, if and only if $\mathscr{J}_{\Omega, D}^{\prime}(W)=0$.

If $\phi$ is a minimal immersion, and $Z$ is a Killing vector field of $\bar{M}$, it is well known that $Z^{\perp}$ is a Jacobi field for the usual Jacobi operator $\left(C_{\Omega}=0\right)$ in the sense that $\mathscr{J}\left(Z^{\perp}\right)=0$ [22]. A proof can be obtained by recalling that Killing vector fields generate a oneparameter family of isometries $\Phi_{t}$ on $\bar{M}$, defining a variation $\phi_{t}=\Phi_{t} \circ \phi$ by minimal immersions, and so a Jacobi field with vector variation. This is also true if $\phi$ has constant mean curvature with $\bar{M}=M^{\prime}, n=1$ and $\Omega$ is the volume form of $M^{\prime}$ [3]. In higher codimension we need some additional assumptions.

Proposition 4.3. If $\phi: M \rightarrow \bar{M}$ is any immersion and $Z$ a Killing vector field of $\bar{M}$, then

$$
\mathscr{J}_{\Omega}\left(Z^{\perp}\right)=\nabla_{Z^{\top}}^{\perp} m H-\left(\bar{\nabla}_{m H} Z\right)^{\perp}+m\|H\| C_{\Omega}\left(Z^{\perp}\right) .
$$

Furthermore, suppose $\phi$ has extended calibrated tangent space and a minimal calibrated extension $M^{\prime}$ of $M$ exists such that $M$ is a closed hypersurface in $M^{\prime}$ as the boundary of 
an open domain $O^{\prime}$ of $M^{\prime}$. Then $\int_{M} a_{Z^{\perp}} d M=0$. In this case, if $\phi$ has parallel mean curvature, then $Z^{\perp}$ is an $\Omega$-Jacobi field along $\phi$ if and only if

$$
-\left(\bar{\nabla}_{m H} Z\right)^{\perp}+m\|H\| C_{\Omega}\left(Z^{\perp}\right)=c v,
$$

where $c=m\|H\| \bar{g}\left(C_{\Omega}\left(Z^{\perp}\right), v\right)$ is a constant, which is zero if $\bar{\nabla} \Omega=0$.

Remark 4.2. (1) If $n=1$, then $H$ is parallel, and $\left(\bar{\nabla}_{H} Z\right)^{\perp}=\|H\| \bar{g}\left(\bar{\nabla}_{v} Z, v\right) v=0$, since $Z$ is Killing. If $C_{\Omega}=0$ (for example $\Omega=d \bar{M}$ ) then $\mathscr{J}_{\Omega}\left(Z^{\perp}\right)=0$.

Proof. Let $p_{0} \in M$ and $e_{i}, W_{\alpha}$ local o.n. frames of $T M$ and $N M$, defined on an open set $D$ of $M$ which contains $p_{0}$, and such that $\nabla_{X} e_{i}\left(p_{0}\right)=\nabla_{X}^{\perp} W_{\alpha}\left(p_{0}\right)=0$, for all $X \in T_{p_{0}} M$. A tubular neighbourhood $\mathscr{V}$ of $D$ in $\bar{M}$ is diffeomorphic to an open set of $N M$, using the exponential map of $\bar{M}$. Each point $q \in \mathscr{V}$ is of the form $q=\exp _{p}(v)$ for a unique $p \in D$ and $v \in N M_{p}$. Let $\gamma(t)$ be the geodesic starting at $p$ with initial velocity $v$. Then we define $\bar{e}_{i}(q)$ and $\bar{W}_{\alpha}(q)$ as the parallel transport along $\gamma(t)$ of $e_{i}(p)$ and of $W_{\alpha}(p)$, respectively. In this way we have vector fields on $\bar{M}$ defined on a neighbourhood of $p_{0}$, extending $e_{i}$ and $W_{\alpha}$. At $p_{0}$ we have

$$
\begin{aligned}
\left(\bar{\nabla}_{e_{i}} W_{\alpha}\right)^{\top} & =-\sum_{j} \bar{g}\left(W_{\alpha}, B\left(e_{i}, e_{j}\right)\right) e_{j}, \quad \bar{\nabla}_{W_{\beta}} \bar{e}_{i}=\bar{\nabla}_{W_{\beta}} \bar{W}_{\alpha}=0 \\
\sum_{i} B\left(e_{i}, \nabla_{e_{i}} Z^{\top}\right) & =\sum_{i j} B\left(e_{i}, e_{j}\right) g\left(e_{j}, \bar{\nabla}_{e_{i}} Z^{\top}\right) \\
& =\sum_{i j} B\left(e_{i}, e_{j}\right) \bar{g}\left(d \phi\left(e_{j}\right), \bar{\nabla}_{e_{i}} Z\right)-\sum_{i j} B\left(e_{i}, e_{j}\right) \bar{g}\left(d \phi\left(e_{j}\right), \bar{\nabla}_{e_{i}} Z^{\perp}\right) \\
& =\sum_{i j} B\left(e_{i}, e_{j}\right) \bar{g}\left(B\left(e_{i}, e_{j}\right), Z^{\perp}\right)=\tilde{B}\left(Z^{\perp}\right),
\end{aligned}
$$

where in the last equality we have used the fact that $B\left(e_{i}, e_{j}\right)$ is symmetric and $\bar{g}\left(\bar{\nabla}_{e_{i}} Z, e_{j}\right)$ skew-symmetric in $i j$. Now we have at $p_{0}$ (and identifying $e_{i}$ with $d \phi\left(e_{i}\right)$ ),

$$
\begin{aligned}
\Delta^{\perp} Z^{\perp} & =\sum_{i \alpha}\left(\bar{\nabla}_{e_{i}}\left(\bar{g}\left(\bar{\nabla}_{e_{i}} Z^{\perp}, W_{\alpha}\right) W_{\alpha}\right)\right)^{\perp}=\sum_{i \alpha}\left(d_{e_{i}} \bar{g}\left(\bar{\nabla}_{e_{i}} Z^{\perp}, W_{\alpha}\right)\right) W_{\alpha} \\
& =\sum_{i \alpha}\left(d_{e_{i}}\left(\bar{g}\left(\bar{\nabla}_{e_{i}} Z, W_{\alpha}\right)-\bar{g}\left(\bar{\nabla}_{e_{i}} Z^{\top}, W_{\alpha}\right)\right)\right) W_{\alpha} \\
& =\sum_{i \alpha}\left(d_{e_{i}}\left(-\bar{g}\left(\bar{\nabla}_{\bar{W}_{\alpha}} Z, e_{i}\right)-\bar{g}\left(B\left(e_{i}, Z^{\top}\right), W_{\alpha}\right)\right)\right) W_{\alpha} \\
& =\sum_{i \alpha}\left(-\bar{g}\left(\bar{\nabla}_{e_{i}} \bar{\nabla}_{\bar{W}_{\alpha}} Z, e_{i}\right)-\bar{g}\left(\bar{\nabla}_{\bar{W}_{\alpha}} Z, \bar{\nabla}_{e_{i}} e_{i}\right)-\bar{g}\left(\nabla_{e_{i}}^{\perp}\left(B\left(e_{i}, Z^{\top}\right)\right), W_{\alpha}\right)\right) W_{\alpha} .
\end{aligned}
$$

Note that for $X, Y$ vector fields on $\bar{M}, \bar{g}\left(\bar{\nabla}_{X} \bar{\nabla}_{Y} Z, Y\right)=0$. Therefore $\bar{g}\left(\bar{\nabla}_{e_{i}} \bar{\nabla}_{\bar{W}_{\alpha}} Z, e_{i}\right)=$ $\bar{g}\left(\bar{R}\left(W_{\alpha}, e_{i}\right) Z+\bar{\nabla}_{\left[\bar{W}_{\alpha}, \bar{e}_{i}\right]} Z, e_{i}\right)$. Using the vanishing properties of the covariant derivatives 
of $e_{i}$ and $W_{\alpha}$ at $p_{0}$, and the fact that $B\left(e_{i}, e_{j}\right)$ is symmetric and $\bar{g}\left(\bar{\nabla}_{e_{i}} Z, e_{j}\right)$ is skewsymmetric in $i j$, we have $\sum_{i} \bar{g}\left(\bar{\nabla}_{\left[\bar{W}_{\alpha}, \bar{e}_{i}\right]} Z, e_{i}\right)=\sum_{i}-\bar{g}\left(\left(\bar{\nabla}_{e_{i}} Z\right)^{\top}, \sum_{j} \bar{g}\left(W_{\alpha}, B\left(e_{j}, e_{i}\right)\right) e_{j}\right)=$ 0. Applying Coddazzi's equation, $\sum_{i} \nabla_{e_{i}}^{\perp}\left(B\left(e_{i}, Z^{\top}\right)\right)=\sum_{i} \nabla_{Z^{\top}} B\left(e_{i}, e_{i}\right)-\left(\bar{R}\left(e_{i}, Z^{\perp}\right) e_{i}\right)^{\perp}+$ $B\left(e_{i}, \nabla_{e_{i}} Z^{\top}\right)$, and we arrive at

$$
\Delta^{\perp} Z^{\perp}=\sum_{i}-\left(\bar{R}\left(e_{i}, Z\right) e_{i}\right)^{\perp}+\left(\bar{\nabla}_{m H} Z\right)^{\perp}-\nabla_{Z^{\top}}^{\perp} H+\sum_{i}\left(\bar{R}\left(e_{i}, Z^{\top}\right) e_{i}\right)^{\perp}-\tilde{B}\left(Z^{\perp}\right) .
$$

Then the expression of $\mathscr{J}_{\Omega}\left(Z^{\perp}\right)$ follows immediately. Now we suppose a calibrated extension $M^{\prime}$ exists with $T M^{\prime}=E M$ along $M$, and $M^{\prime}$ is minimal. Let $Z^{\prime}$ and $Z^{\prime \prime}$ be the projection of $Z$ onto $T M^{\prime}$ and $T M^{\prime \perp}$, respectively. Then for $e_{i}^{\prime}$ a local o.n. frame of $M^{\prime}$,

$$
\operatorname{div}_{M^{\prime}}\left(Z^{\prime}\right)=\sum_{i} \bar{g}\left(\bar{\nabla}_{e_{i}^{\prime}} Z^{\prime}, e_{i}^{\prime}\right)=\sum_{i}-\bar{g}\left(\bar{\nabla}_{e_{i}^{\prime}} Z^{\prime \prime}, e_{i}^{\prime}\right)=(m+1) \bar{g}\left(Z^{\prime \prime}, H^{M^{\prime}}\right)=0,
$$

where $H^{M^{\prime}}$ is the mean curvature of $M^{\prime}$ on $\bar{M}$. Thus, for $M=\partial O^{\prime}$,

$$
\int_{M} a_{Z^{\perp}} d M=\int_{\partial O^{\prime}} \bar{g}\left(Z^{\prime}, v\right)=\int_{O^{\prime}} \operatorname{div}_{M^{\prime}}\left(Z^{\prime}\right)=0 .
$$

By Proposition 4.1, $Z^{\perp} \in H_{T}^{1}(N M)$, and supposing $\nabla^{\perp} H=0$, then Proposition 4.2 yields the equivalence between (14) and the assumption of $Z^{\perp}$ being an $\Omega$-Jacobi field. As $\bar{g}\left(\bar{\nabla}_{v} Z, v\right)=0$ holds, then $c=m\|H\| \bar{g}\left(C_{\Omega}\left(Z^{\perp}\right), v\right)$. If $\Omega$ is parallel, then $c=0$ as explained before Lemma 3.1 .

Remark 4.3. If a normal section $W$ is a solution of the $\Omega$-Jacobi operator $\mathscr{J}_{\Omega, D}^{\prime}(W)=0$, on a compact domain $D$, then $\mathscr{J}_{\Omega}(W)=c v$, where $c=\Psi_{\Omega, D}(W)$ is constant. Supposing $W=0$ on a non-empty open set $D^{\prime} \subset D$, then $\mathscr{J}_{\Omega}(W)=0$ on $D^{\prime}$. Consequently, $c=0$, and $\mathscr{J}_{\Omega}(W)=\mathscr{J}_{\Omega, D}^{\prime}(W)=0$ on $D$. This implies that $\left\|\Delta^{\perp} W\right\| \leq C\left(\|W\|+\left\|\nabla^{\perp} W\right\|\right)$, for some constant $C>0$ depending on $D, \bar{R}, B, \Omega, \bar{\nabla} \Omega$, and $\nabla^{\perp} W_{\alpha}^{\prime}$, where $W_{\alpha}^{\prime}$ is a fixed family of o.n. frames of $N M$ defined on a finite cover of $D$ by compact domains. Thus, by Aronszajn's unique continuation theorem for systems of inequalities of second order (Remark 3 of [1]), $W$ must vanish on all $D$. That is, $\mathscr{J}_{\Omega, D}^{\prime}$ has uniqueness in the Cauchy problem. The extra term $C_{\Omega}$ can be seen to act in the sense of distributions. The coerciveness property associated with $Q_{\Omega}$ still holds on compact domains. To see this we only have to observe that if $P$ is a bilinear map, then $\left|P\left(W, \nabla_{X}^{\perp} W^{\prime}\right)\right| \leq\|P\|(\lambda\|W\|+$ $\left.\lambda^{-1}\left\|\nabla_{X}^{\perp} W^{\prime}\right\|\right)$ holds for any $\lambda>0$, which should be taken sufficiently large (see Chap. 8 [14]). It follows that a Morse index theorem can be stated for submanifolds with parallel mean curvature and calibrated extended tangent spaces by using the $\Omega$-Jacobi fields, in a similar way as Simons's version for minimal submanifolds in [22] (see also [13]).

If a calibrated extension $M^{\prime}$ of $\phi$ exists and $\nabla^{\perp} v=0, M$ has parallel mean curvature in $M^{\prime}$ if and only if it does in $\bar{M}$. If we consider variations $\phi_{t}$ with values on $M^{\prime}$ only, the 
concepts of volume-preserving coincide, for $\Omega$ is the volume form on $M^{\prime}$. In particular, if $f \in \mathscr{F}_{D}$, we have

$$
\begin{aligned}
J_{D}^{\prime \prime}(0)(f) & =\int_{D}-f \Delta f-\left(R^{\prime}+\left\|B^{v}\right\|^{2}\right) f^{2} d M=: I(f, f), \\
& =\int_{D}\|\nabla f\|^{2}-\left(R^{\prime}+\left\|B^{v}\right\|^{2}\right) f^{2} d M=: q(f),
\end{aligned}
$$

where $R^{\prime}=\sum_{i} R^{\prime}\left(e_{i}, v, e_{i}, v\right)=\operatorname{Ricci}^{\prime}(v, v)$, with Ricci' the Ricci tensor of $M^{\prime}$, and $I$ is the bilinear form defined in [3]. The immersion into $M^{\prime}, \phi: D \rightarrow M^{\prime}$, is said to be stable, if $I(f, f) \geq 0$ for all $f \in \mathscr{F}_{D}$. Considering any section $W \in \overline{\mathscr{F}} D, \Omega$ with $W^{\perp}=f v$, then $a_{W}=\bar{g}(W, v)=f$, and using the Gauss equation for $M^{\prime}$ as a submanifold of $\bar{M}$, we have

$$
\begin{aligned}
I_{\Omega}(W, W)= & I(f, f)+m\|H\| \int_{D} f^{2} \bar{g}\left(C_{\Omega}(v), v\right) d M \\
& +\int_{D} f^{2}\left((m+1) \bar{g}\left(H^{M^{\prime}}, B^{M^{\prime}}(v, v)\right)-\left\|B^{M^{\prime}}(v, v)\right\|^{2}\right) d M
\end{aligned}
$$

where $B^{M^{\prime}}$ and $H^{M^{\prime}}$ stand for the second fundamental form and mean curvature of $M^{\prime}$ in $\bar{M}$, respectively. We have used that $B^{M^{\prime}}\left(e_{i}, v\right)=\nabla_{e_{i}}^{\perp} v=0$. Recall the first eigenvalue of the twisted Dirichlet problem [4] (see also a Euclidean version [12]) is given by

$$
\lambda_{\mathscr{F}}(D)=\inf \left\{\frac{q(f)}{\int_{D} f^{2} d M}: \quad f \in \mathscr{F}_{D}\right\} .
$$

Now we consider the case $\bar{g}\left(H^{M^{\prime}}, B^{M^{\prime}}(v, v)\right)=0$ ( for instance, when $M^{\prime}$ is minimal, or $\left.B^{M^{\prime}}(v, v)=0\right)$. We have an orthogonal split $N M=\mathbb{R} v \oplus F$ into two parallel subbundles. If $W \in \mathbb{R} \mathscr{F}_{D, \Omega}, W=W^{v}+W^{F}=f v+W^{F}$, where $f \in \mathscr{F}_{D}$ and $W^{F} \in \mathscr{F}^{\prime}(F)$, then

$$
\begin{gathered}
I_{\Omega}(W, W)=I(f, f)+I_{\Omega}\left(W^{F}, W^{F}\right)-\int_{D} f^{2}\left(\left\|B^{M^{\prime}}(v, v)\right\|^{2}-m\|H\| \bar{g}\left(C_{\Omega}(v), v\right)\right) d M \\
-2 \int_{D} f\left(\sum_{i} \bar{R}\left(e_{i}, v, e_{i}, W^{F}\right)+\sum_{i j} B^{v}\left(e_{i}, e_{j}\right) \bar{g}\left(B\left(e_{i}, e_{j}\right), W^{F}\right)\right) d M .
\end{gathered}
$$

There are several situations with $C_{\Omega}=0$. One is given in Proposition 3.2. Another is when $n=2$ and $\bar{\nabla} \Omega=0$. In Lemma 4.4 we will completely characterize this condition.

Theorem 4.1. We suppose a calibrated extension $M^{\prime}$ of $M$ exists satisfying the condition $\bar{g}\left(H^{M^{\prime}}, B^{M^{\prime}}(v, v)\right)=0$.

(1) If $\phi: M \rightarrow \bar{M}$ is $\Omega$-stable on $D$, then $\phi: M \rightarrow M^{\prime}$ is also stable on $D$ and

$$
\lambda_{\mathscr{F}}(D) \geq \inf _{D}\left(\left\|B^{M^{\prime}}(v, v)\right\|^{2}-m\|H\| \bar{g}\left(C_{\Omega}(v), v\right)\right) .
$$


(2) If $M^{\prime}$ is a totally geodesic submanifold of $\bar{M}$ and $C_{\Omega}=0$, we have for $W \in \mathscr{F} D, \mathscr{F}$

$$
I_{\Omega}(W, W)=I(f, f)+I_{\Omega}\left(W^{F}, W^{F}\right) .
$$

In the particular case $\bar{g}\left(\bar{R}\left(W^{F}\right), W^{F}\right) \leq 0$, we have $I_{\Omega}(W, W) \geq I(f, f)+\int_{D}\left\|\nabla^{\perp} W^{F}\right\|^{2}$. In this case, if $\phi: M \rightarrow M^{\prime}$ is stable, then $\phi: M \rightarrow \bar{M}$ is also $\Omega$-stable.

Proof. It is clear that if $\phi: M \rightarrow \bar{M}$ is $\Omega$-stable on $D$ then $\phi: M \rightarrow M^{\prime}$ is also stable on $D$. By the assumptions, (15) reads, for $W^{\perp}=f \nu$ with $f \in \mathscr{F}_{D}$,

$$
I_{\Omega}(W, W)=I(f, f)-\int_{D} f^{2}\left(\left\|B^{M^{\prime}}(v, v)\right\|^{2}-m\|H\| \bar{g}\left(C_{\Omega}(v), v\right)\right) d M .
$$

Take $f \in \mathscr{F}_{D}$ an eigenvector of $\lambda_{\mathscr{F}}=\lambda_{\mathscr{F}}(D)$ for the associated twisted Dirichlet problem on $D$, that is, $-\Delta f-\left(R^{\prime}+\left\|B^{v}\right\|\right) f=\lambda_{\mathscr{F}} f+\Psi(f)$, where $\Psi(f)=|D|^{-1} \int_{D}(-\Delta f-$ $\left.\left(R^{\prime}+\left\|B^{v}\right\|\right) f\right)$. Then $I(f, f)=\lambda_{\mathscr{F}} \int_{D} f^{2}$. From (17) and the fact that $I_{\Omega}(W, W) \geq 0$, (1) follows immediately. If $M^{\prime}$ is totally geodesic, then $B\left(e_{i}, e_{j}\right)=B^{v}\left(e_{i}, e_{j}\right)$ takes values on $T M^{\prime}$, as well as $\bar{R}\left(e_{i}, v\right) e_{i}=R^{\prime}\left(e_{i}, v\right) e_{i}$. Thus the last terms of (16) vanish. Moreover $\tilde{B}\left(W^{F}\right)=0$. Consequently $I_{\Omega}\left(W^{F}, W^{F}\right)=\int_{D}\left(\left\|\nabla^{\perp} W^{F}\right\|^{2}-\bar{g}\left(\bar{R}\left(W^{F}\right), W^{F}\right)\right) d M$. If $\bar{g}\left(\bar{R}\left(W^{F}\right), W^{F}\right) \leq 0$, then $I_{\Omega}\left(W^{F}, W^{F}\right) \geq \int_{D}\left\|\nabla^{\perp} W^{F}\right\|^{2} d M$, which proves (2).

Barbosa, do Carmo and Eschenburg proved in [3] that geodesic spheres of space forms are the unique stable hypersurfaces of constant mean curvature. The uniqueness is established by showing that the stability condition implies the hypersurface to be umbilical. As an immediate consequence of this result and the preceding theorem, we have:

Corollary 4.1. If a calibrated extension $M^{\prime}$ exits, and is a space form, if $\phi: M \rightarrow \bar{M}$ is $\Omega$-stable then $M$ is a geodesic sphere of $M^{\prime}$.

In the general case the calibrated extension $M^{\prime}$ is not a space form, and so geodesic spheres of $M^{\prime}$ may have no constant mean curvature, nor be umbilical (the second fundamental form of geodesic spheres is, up to a sign, the Hessian of the distance function to a point), but umbilical submanifolds may exist. Furthermore, geodesic $m$-spheres in a Euclidean space or in a Euclidean $(m+n)$-sphere with $n \geq 2$ may not be stable (see Propositions 4.5 and 4.6(2)). For the case of positive sectional curvature, a more general statement is the following:

Proposition 4.4. Assume $M$ is closed, and $\bar{\nabla} \Omega=0$ or $C_{\Omega}=0$. If $N M$ allows a global unit parallel section $v^{F}$ orthogonal to $v$, and if $\int_{M} \sum_{i} \bar{R}\left(e_{i}, v^{F}, e_{i}, v^{F}\right) d M>0$, then $M$ is $\Omega$-unstable. 
Proof. For $\bar{\nabla} \Omega=0$ and $\nabla^{\perp} v^{F}=0$ we also have $\bar{g}\left(C_{\Omega}\left(v^{F}\right), v^{F}\right)=0$. By Proposition $4.1 v^{F}$ is in the extended domain of $I_{\Omega}$, and $I_{\Omega}\left(v^{F}, v^{F}\right)=-\int_{M} \sum_{i} \bar{R}\left(v^{F}, e_{i}, v^{F}, e_{i}\right)+$ $\sum_{i j}\left(\bar{g}\left(B\left(e_{i}, e_{j}\right), v^{F}\right)\right)^{2}<0$.

We cannot expect Euclidean spheres to be $\Omega$-stable in $\mathbb{R}^{m+n}$ for any calibration $\Omega$. We will show in the next proposition how stability depends on $\Omega$. For any submanifold $M$, consider the tensor $\xi: \wedge^{2} N M \rightarrow T M^{*}$ defined by

$$
\xi\left(W, W^{\prime}\right)(u)=\Omega\left(W, W^{\prime}, * u\right) \quad(\text { with } * \text { the star operator on } M) .
$$

Lemma 4.4. The differential operator $C_{\Omega}$ vanishes if and only if $\xi$ vanishes and,

$$
\bar{\nabla}_{W} \Omega\left(W^{\prime}, d \phi\left(e_{1}\right), \ldots, d \phi\left(e_{m}\right)\right)=-\bar{\nabla}_{W^{\prime}} \Omega\left(W, d \phi\left(e_{1}\right), \ldots, d \phi\left(e_{m}\right)\right) \quad \forall W, W^{\prime} \in N M .
$$

Proof. Fixing a point $p \in M$ and $W_{a}$ a local o.n. frame of $N M$ that satisfies $\nabla^{\perp} W_{a}(p)=0$, we see that $\bar{g}\left(C_{\Omega}\left(W_{a}\right), W_{b}\right)=0$ is equivalent to the last condition, and taking $W=$ $W_{a}+f W_{b}$ where $f$ is any local function, the condition $\bar{g}\left(C_{\Omega}(W), W\right)=0$, at $p$, translates into $\xi\left(W_{a}, W_{b}\right)(\nabla f)=0$, at $p$. Since $\nabla f(p)$ is arbitrary, we conclude $\xi=0$.

Proposition 4.5. Suppose $M$ is a closed pseudo-umbilical submanifold of $\bar{M}=\mathbb{R}^{m+n}$, has parallel mean curvature, and calibrated extended tangent space. We also suppose $\Omega$ is a calibration on $\mathbb{R}^{m+n}$ that satisfies $\bar{\nabla}_{W} \Omega\left(W, e_{1}, \ldots, e_{m}\right)=0$ for any $W \in N M$. Let $M^{\prime}$ be the minimal $\Omega$-calibrated extension given in Example 2.4, and $\lambda_{1}$ be the first non-zero eigenvalue of $M$ for the closed eigenvalue problem.

(1) $M$ is stable in $M^{\prime}$ if and only if $\lambda_{1} \geq m\|H\|^{2}$. This holds (with equality) when $M^{\prime}=$ $\mathbb{R}^{m+1}$.

(2) Assume $M$ is totally umbilical in $\bar{M}$, that is, $M^{\prime}$ is an $(m+1)$-Euclidean space and $M$ is a Euclidean m-sphere of $M^{\prime}$, and fix a global parallel basis $W_{\alpha}$ of $T M^{\prime \perp}=\mathbb{R}^{n-1}$. Then, $M$ is $\Omega$-stable in $\bar{M}$ if and only if the 1 -forms $\xi\left(W_{\alpha}, W_{\beta}\right)$ are co-exact, that is, $\xi\left(W_{\alpha}, W_{\beta}\right)=\delta \omega_{\alpha \beta}$, for some 2 -forms $\omega_{\alpha \beta}$ on $M$, and they satisfy the inequality

$$
\sum_{\alpha<\beta}-2 m\|H\| \int_{M}\left\langle\omega_{\alpha \beta}, d f_{\alpha} \wedge d f_{\beta}\right\rangle d M \leq \sum_{\alpha} \int_{M}\left\|d f_{\alpha}\right\|^{2} d M \quad \forall f_{\alpha}, f_{\beta} \in C^{\infty}(M)
$$

where $\langle$,$\rangle denotes the usual Hilbert-Schmidt inner product for 2-forms. In this case, for$ each $\alpha, \beta$ the following estimates for $\omega_{\alpha \beta}$ holds, viz.

$$
\begin{aligned}
& 2 m\|H\|\left|\int_{M}\left\langle\omega_{\alpha \beta}, d f \wedge d h\right\rangle d M\right| \leq \int_{M}\left(\|d f\|^{2}+\|d h\|^{2}\right) d M, \\
& m\|H\|\left|\int_{M} \omega_{\alpha \beta}(\nabla f, \nabla h) d M\right| \leq \sqrt{\int_{M}\|\nabla f\|^{2} d M} \sqrt{\int_{M}\|\nabla h\|^{2} d M},
\end{aligned}
$$

for any smooth functions $f, h$. Furthermore, if $C_{\Omega}=0$, then $M$ is $\Omega$-stable in $\bar{M}$. 
Proof. (1) Recall that for $M$ closed, $\lambda_{1}=\inf _{f \in \mathscr{F}_{M}}\left(\int_{M}\|\nabla f\|^{2}\right) /\left(\int_{M} f^{2}\right)$. Since $\left\|B^{v}\right\|^{2}=$ $m\|H\|^{2}$ and by Proposition 2.1 in Example 2.4, $R^{\prime}=\operatorname{Ricci}^{\prime}(v, v)=-\left\|B^{M^{\prime}}(v, v)\right\|^{2}=0$, then $q(f)=\int_{M}\|\nabla f\|^{2} d M-m\|H\| \int_{M} f^{2} d M$, and (1) follows from the above Reighley characterization of $\lambda_{1}$. (2) If $M$ is umbilical in $\bar{M}, M$ is a sphere by Proposition 2.1. From the assumptions, $\bar{g}\left(C_{\Omega}(v), v\right)=0$. Then (16) gives us $I_{\Omega}(W, W)=I(f, f)+I_{\Omega}\left(W^{F}, W^{F}\right)$, with $I_{\Omega}\left(W^{F}, W^{F}\right)=\int_{M}\left(\left\|\nabla^{\perp} W^{F}\right\|^{2}+m\|H\| \bar{g}\left(C_{\Omega}\left(W^{F}\right), W^{F}\right)\right) d M$. Since $\lambda_{1}=m\|H\|^{2}$, then by (1), $I(f, f) \geq 0$, and equality holds for $f$ an eigenfunction of $\lambda_{1}$. Thus, $M$ is $\Omega$-stable if and only if $I_{\Omega}\left(W^{F}, W^{F}\right) \geq 0$. We take $W_{\alpha}$ a global o.n. frame of parallel sections of $T M^{\prime \perp}=\mathbb{R}^{n-1}$, and set $W^{F}=\sum_{\alpha} f_{\alpha} W_{\alpha}$, with $f_{\alpha}$ arbitrary functions. Note that $* e_{i}=(-1)^{i-1} e_{1} \wedge \ldots \wedge \hat{e}_{i} \wedge \ldots \wedge e_{m}$. Then, the $\Omega$-stability condition translates into

$$
\int_{M}\left(\sum_{\alpha}\left\|\nabla f_{\alpha}\right\|^{2}+\sum_{\alpha \beta} m\|H\| f_{\alpha} \xi\left(W_{\alpha}, W_{\beta}\right)\left(\nabla f_{\beta}\right)\right) d M \geq 0 .
$$

Now we prove the $\xi\left(W_{\alpha}, W_{\beta}\right)$ are co-closed. If we choose $f_{\alpha}=1$ and $f_{\beta}$ arbitrary, and $f_{\gamma}=0$ for $\gamma \neq \alpha, \beta$, we get from (19)

$$
\int_{M}\left(\left\|\nabla f_{\beta}\right\|^{2}+m\|H\| \xi\left(W_{\alpha}, W_{\beta}\right)\left(\nabla f_{\beta}\right)\right) d M \geq 0 .
$$

Replacing $f_{\beta}$ by $t f_{\beta}$, with $t>0$ a constant, and letting $t \rightarrow 0$, we obtain $\int_{M} \xi\left(W_{\alpha}, W_{\beta}\right)\left(\nabla f_{\beta}\right)$ $\geq 0$, and again, replacing $f_{\beta}$ by $-f_{\beta}$, we obtain equality to zero. Since we have $\xi\left(W_{\alpha}, W_{\beta}\right)\left(\nabla f_{\beta}\right)=\left\langle\xi\left(W_{\alpha}, W_{\beta}\right), d f_{\beta}\right\rangle$, we conclude that $\xi\left(W_{\alpha}, W_{\beta}\right)$ are $L^{2}$-orthogonal to all exact 1-forms $d f_{\beta}$ on $M$. As the Betti numbers of the spheres vanish, by the Hodge decomposition theorem $\xi\left(W_{\alpha}, W_{\beta}\right)$ are co-exact, that is, $\xi\left(W_{\alpha}, W_{\beta}\right)=\delta \omega_{\alpha \beta}$, for some 2 -forms $\omega_{\alpha \beta}$. Then inequality (19) is equivalent to

$$
\int_{M} \sum_{\alpha}\left|d f_{\alpha}\right|^{2} d M+\sum_{\alpha \beta} m\|H\| \int_{M}\left\langle\omega_{\alpha \beta}, d f_{\alpha} \wedge d f_{\beta}\right\rangle d M \geq 0,
$$

which gives the first inequality of the proposition. Fixing $\alpha<\beta$ and setting $f=f_{\alpha}$, $h=f_{\beta}$, and $f_{\gamma}=0$ for $\gamma \neq \alpha, \beta$, the above inequality implies

$$
\int_{M}\left(|d f|^{2}+|d h|^{2}\right) d M+2 m\|H\| \int_{M}\left\langle\omega_{\alpha \beta}, d f \wedge d h\right\rangle d M \geq 0 .
$$

If we change $f$ by $-f$, we conclude the second inequality of the proposition. Note that $\left\langle\omega_{\alpha \beta}, d f \wedge d h\right\rangle=\omega_{\alpha \beta}(\nabla f, \nabla h)$. The last inequality is obtained from the second one by multiplying $f$ by a constant $t$ and $h$ by $t^{-1}$, with $t^{2}=\|\nabla h\|_{L^{2}} /\|\nabla f\|_{L^{2}}$. Finally, if $C_{\Omega}=0$, by Lemma 4.4, $\xi\left(W_{\alpha}, W_{\beta}\right)=0$.

We note that for any parallel calibration $\Omega$ of $\mathbb{R}^{m+n}$, and any sphere $M$ of a calibrated 
vector space $\mathbb{R}^{m+1}$, if we fix $W_{\alpha}$ a constant o.n. frame of $T M^{\perp \perp}=\mathbb{R}^{n-1}$, then the $(m-1)$ forms $\hat{\xi}_{\alpha \beta}=\Omega\left(W_{\alpha}, W_{\beta}, \cdots\right)$ are parallel in $\mathbb{R}^{m+n}$. We may take in previous proposition $\xi\left(W_{\alpha}, W_{\beta}\right)=* \phi^{*} \hat{\xi}_{\alpha \beta}$, that are obviously co-closed on $M$. Many well known calibrations in $\mathbb{R}^{m+n}$ satisfy $C_{\Omega} \neq 0$ with $\xi\left(W_{\alpha}, W_{\beta}\right)$ co-closed. On the other hand, to investigate if inequalities in Proposition 4.5(2) are satisfied or not seems to be not so easy to determine, as we can see in next remark.

Remark 4.4. (1) The associative calibration of $\mathbb{R}^{7}$ is the 3 -form given by

$$
\Omega=\varepsilon_{*}^{123}+\varepsilon_{*}^{145}+\varepsilon_{*}^{167}+\varepsilon_{*}^{246}-\varepsilon_{*}^{257}-\varepsilon_{*}^{347}-\varepsilon_{*}^{356}
$$

where $\varepsilon_{i}$ is the canonical basis of $\mathbb{R}^{7}$. We are considering $\mathbb{S}^{2}$ the unit sphere of the calibrated subspace spanned by $\varepsilon_{i}, i=1,2,3$ and $W_{\alpha}=\varepsilon_{\alpha}, \alpha=4,5,6,7$. Then we have $\hat{\xi}_{45}=\hat{\xi}_{67}=\varepsilon_{*}^{1}=d x^{1}, \hat{\xi}_{46}=-\hat{\xi}_{57}=\varepsilon_{*}^{2}=d x^{2}, \hat{\xi}_{47}=-\hat{\xi}_{56}=-\varepsilon_{*}^{3}=-d x^{3}$. Consequently, $\omega_{\alpha \beta}=\rho_{\alpha \beta} \operatorname{Vol}_{\mathbb{S}^{2}}$ with $\rho_{45}=\rho_{67}=-\phi^{1}, \rho_{46}=-\rho_{57}=-\phi^{2}, \rho_{47}=-\rho_{56}=\phi^{3}$, where $\phi: \mathbb{S}^{2} \rightarrow \mathbb{R}^{3} \subset \mathbb{R}^{7}$ is the inclusion map. Let us suppose that $\phi$ is $\Omega$-stable. Then $\omega_{45}$ should satisfy the last inequality of Proposition 4.5 , i.e. for any functions $f, h: \mathbb{S}^{2} \rightarrow \mathbb{R}$

$$
2\left|\int_{\mathbb{S}^{2}} \phi^{1} \operatorname{Vol}_{\mathbb{S}^{2}}(\nabla f, \nabla h) d M\right| \leq \sqrt{\int_{\mathbb{S}^{2}}\|\nabla f\|^{2} d M} \sqrt{\int_{\mathbb{S}^{2}}\|\nabla h\|^{2} d M}
$$

We now use the stereographic projection $\sigma: \mathbb{R}^{2} \rightarrow \mathbb{S}^{2} \subset \mathbb{R}^{3}, \sigma(w)=\left(\frac{\left(|w|^{2}-1\right)}{\left(|w|^{2}+1\right)}, \frac{2 w}{\left(|w|^{2}+1\right)}\right)$, that is a conformal map. We denote by $\mathrm{Vol}_{0}$ the Euclidean volume element of $\mathbb{R}^{2}, J$ the canonical complex structure, and by $\nabla^{0} f$ the Euclidean gradient for a function defined on $\mathbb{R}^{2}$. Then the above inequality is equivalent to

$$
2\left|\int_{\mathbb{R}^{2}}\left(\frac{|w|^{2}-1}{|w|^{2}+1}\right) \operatorname{Vol}_{0}\left(\nabla^{0} f, \nabla^{0} h\right) d w\right| \leq \sqrt{\int_{\mathbb{R}^{2}}\left|\nabla^{0} f\right|^{2} d w} \sqrt{\int_{\mathbb{R}^{2}}\left|\nabla^{0} h\right|^{2} d w}
$$

for functions $f, h: \mathbb{R}^{2} \rightarrow \mathbb{R}$, that we take with compact support in an annulus $D:=\{w:$ $\left.0 \leq R_{1} \leq|w| \leq R_{2}\right\}$. We choose $R_{1}$ sufficiently large so that $\left(|w|^{2}-1\right) /\left(|w|^{2}+1\right) \geq$ $\frac{1}{2}+\delta$, where $0<\delta<1 / 2$ is a constant. Since $g_{0}\left(J \nabla^{0} f, \nabla^{0} h\right)=\operatorname{Vol}_{0}\left(\nabla^{0} f, \nabla^{0} h\right)$, and if this is $\geq 0$, from preceding inequality we have $2\left(\frac{1}{2}+\delta\right)\left|\int_{D} g_{0}\left(J \nabla^{0} f, \nabla^{0} h\right) d w\right| \leq$ $\left|\nabla^{0} f\right|_{L^{2}}\left|\nabla^{0} h\right|_{L^{2}}$. A pair of functions $\left(h, h^{\prime}\right)$ on $D$ defines a holomorphic map in $\mathbb{C}$ if and only if $-J \nabla^{0} h^{\prime}(w)=\nabla^{0} h(w)$. Thus, we maximize $g_{0}\left(J \nabla^{0} f, \nabla h\right)$ by taking $f=-h^{\prime}$ for such pair of conjugate harmonic maps, giving $\operatorname{Vol}_{0}\left(\nabla^{0} f, \nabla^{0} h\right)=\left\|\nabla^{0} f\right\|\left\|\nabla^{0} h\right\|=$ $\left\|\nabla^{0} f\right\|^{2}$. This would give a contradiction in the previous inequality. As a matter of fact, we cannot choose such a pair of functions, because nonconstant harmonic maps cannot vanish in all $\partial D$. We also recall that a harmonic function $f$ on $\mathbb{R}^{2}$ with $L^{2}$ derivative defines a $L^{2}$-harmonic one-form $d f$, and so, by a result of Yau ( [24], Theorem 6), $f$ must 
be constant. The question is to know how far is an holomorphic map $\left(\varphi, \varphi^{\prime}\right)$ on $D$ from a pair of functions $(f, h)$ vanishing in $\partial D$. This can be measured by $\left|\operatorname{Vol}_{0}\left(\nabla^{0} f, \nabla h\right)\right|$ as we have described. A significant distance between these two set of pairs of functions could indicate that (18) holds. We also observe that $\phi^{k}$ are $\lambda_{1}$-eigenfunctions with $\lambda_{1}=2$ the first nonzero eigenvalue of $\mathbb{S}^{2}$, and they satisfy $\int_{\mathbb{S}^{2}}\left(\phi^{k}\right)^{2} d M=\frac{1}{3}\left|\mathbb{S}^{2}\right|$. Furthermore, the inequality (18) holds if we take $f_{\alpha}$ and $f_{\beta}$ any $\lambda_{i}$-eigenfunctions, with $i=1$ or 2 , giving either equality, or zero in the 1.h.s. The fact that $\omega_{\alpha \beta}$ is defined using the $\lambda_{1}$-eigenfunctions suggests us that a proof of the $\Omega$-stability of the 2 -sphere should be related to some inequalities derived from spectral theory, and this will be the subject of future work.

(2) Let us now consider the Kähler calibration of $\mathbb{R}^{6}$ given by the 4 -form

$$
\Omega=\frac{1}{2}\left(\varepsilon_{*}^{12}+\varepsilon_{*}^{34}+\varepsilon_{*}^{56}\right) \wedge\left(\varepsilon_{*}^{12}+\varepsilon_{*}^{34}+\varepsilon_{*}^{56}\right)=\varepsilon_{*}^{1234}+\varepsilon_{*}^{1256}+\varepsilon_{*}^{3456}
$$

where $\varepsilon_{i}$ is the canonical basis of $\mathbb{R}^{7}$, and $\phi=\left(\phi^{1}, \ldots, \phi^{4}\right): \mathbb{S}^{3} \rightarrow \mathbb{R}^{4}$ denotes the inclusion map of the 3 -sphere of the calibrated subspace $\mathbb{R}^{4}$ spanned by $\varepsilon_{i}, i=1,2,3,4$. Then we are taking $\hat{\xi}_{56}=\varepsilon_{*}^{12}+\varepsilon_{*}^{34}=d x^{1} \wedge d x^{2}+d x^{3} \wedge d x^{4}$. The $\Omega$-stability condition is equivalent to the inequality

$$
3\left|\int_{\mathbb{S}^{3}}\left\langle\omega_{56}, d f_{5} \wedge d f_{6}\right\rangle d M\right| \leq \sqrt{\int_{\mathbb{S}^{3}}\left\|\nabla f_{5}\right\|^{2} d M} \sqrt{\int_{\mathbb{S}^{3}}\left\|\nabla f_{6}\right\|^{2} d M}
$$

to be valid for all smooth maps $f_{5}, f_{6}$ on $\mathbb{S}^{3}$. We have $* \omega_{56}=\phi^{1} d \phi^{2}+\phi^{3} d \phi^{4}$, and $\left\langle\omega_{56}, d f_{5} \wedge d f_{6}\right\rangle d M=d f_{5} \wedge d f_{6} \wedge * \omega_{56}$. If we take $f_{\alpha}$ one of the components $\phi^{i}$ ( that are $\lambda_{1}$-eigenfunctions, with $\lambda_{1}=3$ the first non-zero eigenvalue of $\mathbb{S}^{3}$ ), we can verify, using spherical coordinates, that the previous inequality holds, with equality in some cases. Once more, a proof for stability seems to be related to new spectral inequalities as in preceding case (1).

Next we obtain a uniqueness theorem which extends the case $n=1$ [2]:

Theorem 4.2. Assume that $\bar{M}=\mathbb{R}^{m+n}, \bar{\nabla} \Omega=0$ (or $\left.C_{\Omega}=0\right)$, and $M$ is a closed submanifold with parallel mean curvature and calibrated extended tangent space. Consider the height functions

$$
h=\bar{g}(\phi, v) \quad \text { and } \quad S=\sum_{i j} \bar{g}\left(\phi,\left(B\left(e_{i}, e_{j}\right)\right)^{F}\right) B^{v}\left(e_{i}, e_{j}\right) .
$$

If $\phi: M \rightarrow \bar{M}$ is $\Omega$-stable and $\int_{M} S(2+h\|H\|) d M \leq 0$, then $\phi$ is pseudo-umbilical and a minimal calibrated extension $M^{\prime}$ of $M$ exists with $R^{\prime}=-\left\|B^{M^{\prime}}(v, v)\right\|^{2}=0$ and $S=0$. Furthermore, if $N M$ is a trivial bundle, then $M^{\prime}=\mathbb{R}^{m+1}, M$ is a Euclidean sphere and $\Omega$ satisfies Proposition 4.5(2). 
Proof. If $\phi$ is pseudo-umbilical, then by Proposition 2.1 a (minimal) calibrated extension $M^{\prime}$ exists, satisfying $R^{\prime}=-\left\|B^{M^{\prime}}(v, v)\right\|^{2}=0$, and $S=-\bar{g}\left(B^{M^{\prime}}(v, v), \phi\right) H=0$. We follow [2]. Let $\bar{X}_{x}=x$ be the position vector field in $\mathbb{R}^{m+n}$. Using the well-known expression $m \bar{g}(H, \phi)=\operatorname{div}\left(\phi^{\top}\right)-\frac{1}{2} \operatorname{tr}_{g} L_{\bar{X}} \bar{g}$, where $\phi^{\top}(x)$ is the projection of $\phi(x)$ onto $T_{x} M$, and integrating over $M$, we have

$$
\int_{M}(\|H\| h+1) d M=0 .
$$

That is, $f=(\|H\| h+1) \in \mathscr{F}_{M}$. Now, $d h\left(e_{i}\right)=\bar{g}\left(\phi, d v\left(e_{i}\right)\right)=\sum_{j}-\bar{g}\left(\phi, e_{j}\right) B^{v}\left(e_{j}, e_{i}\right)$. By applying Coddazzi's equation we have $\sum_{i} \nabla_{e_{i}} B^{v}\left(e_{i}, e_{j}\right)=m \bar{g}\left(\nabla_{e_{j}}^{\perp} H, v\right)=0$. We may assume at a given point $\nabla_{e_{i}} e_{j}=0$. Then at that point

$$
\begin{aligned}
\Delta h & =\sum_{i j}-\delta_{i j} B^{v}\left(e_{i}, e_{j}\right)-\bar{g}\left(\phi, B\left(e_{i}, e_{j}\right)\right) B^{v}\left(e_{i}, e_{j}\right)-\bar{g}\left(\phi, e_{j}\right) \nabla_{e_{i}} B^{v}\left(e_{j}, e_{i}\right) \\
& =-m\|H\|-\bar{g}\left(\phi, B\left(e_{i}, e_{j}\right)\right) B^{v}\left(e_{i}, e_{j}\right)=-m\|H\|-h\left\|B^{v}\right\|^{2}-S,
\end{aligned}
$$

and integration over $M$ give us the equality

$$
\int_{M} h\left\|B^{v}\right\|^{2} d M=-\int_{M}(S+m\|H\|) d M .
$$

The stability condition applied to $W=f v$, and the fact that $\bar{g}\left(C_{\Omega}(v), v\right)=0$, by assumptions on $\Omega$ and $v$, implies $\int_{M}-f \Delta f-f^{2}\left\|B^{v}\right\|^{2} \geq 0$, that is,

$$
\begin{aligned}
& \int_{M}\left(\|H\|^{2} h^{2}\left\|B^{v}\right\|^{2}+m\|H\|^{3} h+\|H\|^{2} h S+\|H\| h\left\|B^{v}\right\|^{2}+m\|H\|^{2}+\|H\| S\right) d M \\
& \geq \int_{M}\left(\left\|B^{v}\right\|^{2} \mid H\left\|^{2} h^{2}+2\right\| B^{v}\left\|^{2}\right\| H\|h+\| B^{v} \|^{2}\right) d M,
\end{aligned}
$$

and using the above equalities (20)(21), we get the simplified inequality

$$
\int_{M}\|H\| S(\|H\| h+1) d M \geq \int_{M}\left\|B^{v}\right\|^{2}(\|H\| h+1) d M=\int_{M}\left\|B^{v}\right\|^{2}-\|H\|(S+m\|H\|) d M,
$$

that is, $\int_{M}\|H\| S(\|H\| h+2) d M \geq \int_{M}\left(\left\|B^{v}\right\|^{2}-m\|H\|^{2}\right) d M$. By assumption, and the fact that $\left\|B^{v}\right\|^{2} \geq m\|H\|^{2}$, we get $\left\|B^{v}\right\|^{2}=m\|H\|^{2}$, which proves $M$ is pseudo-umbilical. Thus, $S=0$. If $N M$ is spanned by a global orthonormal system of $n$ parallel sections $\left\{v, W_{\alpha}\right\}$, then $\bar{g}\left(C_{\Omega}\left(W_{\alpha}\right), W_{\alpha}\right)=0$ and the $\Omega$-stability implies

$$
I_{\Omega}\left(W_{\alpha}, W_{\alpha}\right)=-\int_{M} \sum_{i j} \bar{g}\left(B\left(e_{i}, e_{j}\right), W_{\alpha}\right)^{2} \geq 0
$$

that is, $B^{M^{\prime}}\left(e_{i}, e_{j}\right)=0$. Thus $\phi$ is totally umbilical and, by Proposition 2.1, $M$ is a sphere on $M^{\prime}=\mathbb{R}^{m+1}$ and $C_{\Omega}$ satisfies Proposition 4.5(2). 
Now we specialize on the case that the calibration is $\Omega_{\pi}$, defined by a Riemannian fibration $\pi: \bar{M} \rightarrow N$ of totally geodesic fibres. We recall that the Riemannian submersions of the unit Euclidean spheres $\mathbb{S}^{m+n}$, with totally geodesic and connected fibres, were classified by Escobales and Ranjan [10, 20], and define the Hopf fibrations of the spheres. Among these fibrations, the ones that have fibres of dimension $\geq 3$ are the Hopf fibrations of $\mathbb{S}^{7}$ with fibre $\mathbb{S}^{3}$, of $\mathbb{S}^{4 k+3}$, for $k \geq 2$, with fibre $\mathbb{S}^{3}$, and of $\mathbb{S}^{15}$ with fibre $\mathbb{S}^{7}$, i.e.

$$
\mathbb{S}^{3} \hookrightarrow \mathbb{S}^{7} \rightarrow \mathbb{S}^{4}\left(\frac{1}{2}\right), \quad \mathbb{S}^{3} \hookrightarrow \mathbb{S}^{4 k+3} \rightarrow \mathbb{H} \mathbb{P}^{k}, \quad \mathbb{S}^{7} \hookrightarrow \mathbb{S}^{15} \rightarrow \mathbb{S}^{8}\left(\frac{1}{2}\right)
$$

respectively, where $\mathbb{H}^{k}$ is the quaternionic projective space of sectional curvature $K$ with $1 \leq K \leq 4$, and $\mathbb{S}^{4}\left(\frac{1}{2}\right)$ and $\mathbb{S}^{8}\left(\frac{1}{2}\right)$ are spheres of curvature 4 . Fibrations of $\mathbb{H}^{m+2}$ by totally geodesic hypersurfaces (and so by $(m+1)$-dimensional hyperbolic spaces) were described by Ferus [11], and they arise as the nullity foliation of a suitable isometric immersion of $\mathbb{H}^{m+2}$ into $\mathbb{H}^{m+3}$ without umbilics.

Proposition 4.6. (1) Any Euclidean m-dimensional sphere of an $(m+1)$-dimensional

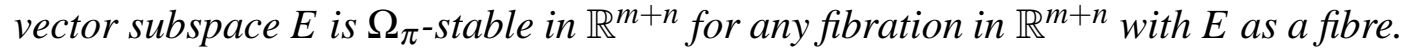

(2) Let $M^{m}$ be a geodesic sphere of $\mathbb{S}^{m+1}$. Immersing $\mathbb{S}^{m+1}$ as a totally geodesic fibre in $\mathbb{S}^{m+n}$, where $m, n$ are such that $\pi: \mathbb{S}^{m+n} \rightarrow N$ is one of the Hopf fibrations given in (22), defines an $\Omega_{\pi}$-unstable immersion $\phi: M^{m} \rightarrow \mathbb{S}^{m+n}$ with parallel mean curvature.

(3) If $M^{m}$ is a geodesic sphere of a hyperbolic space $\mathbb{H}^{m+1}$, with $\mathbb{H}^{m+1}$ immersed as a fibre of a Riemannian fibration $\pi: \mathbb{H}^{m+n} \rightarrow N$ of the $(m+n)$-dimensional hyperbolic space, with $n \geq 2$, and by totally geodesic fibres, then the corresponding immersion $\phi: M^{m} \rightarrow \mathbb{H}^{m+n}$ is an $\Omega_{\pi^{-s t a b l e ~ i m m e r s i o n ~ w i t h ~ p a r a l l e l ~ m e a n ~ c u r v a t u r e . ~}}$

Proof. (1) and (3) are immediate consequences of Theorem 4.1(2) and Proposition 3.2. Now we prove (2). In [3] it is proved that $I(f, f) \geq 0$ for all $f \in \mathscr{F}_{M}$. On the other hand, the normal bundle $N\left(\mathbb{S}^{m+1}\right)$ of $\mathbb{S}^{m+1}$ in $\mathbb{S}^{m+n}$ is a trivial bundle, spanned by $n-1$ unit parallel vector fields $V_{1}, \ldots, V_{n-1}$. Their restrictions to $M$ are parallel along $M$, and they span the parallel subbundle $F$ of $N M$. By Proposition 4.4 with $C_{\Omega}=0$ (see Proposition 3.2), $M$ is $\Omega$-unstable.

Acknowledgements. The author is indebted to Pedro Freitas for his help on handling the twisted eigenvalue problem and related spaces of functions.

\section{References}

[1] Aronszajn, N.: A unique continuation theorem for solutions of elliptic partial differential equations or inequalities of second order. J. Math. Pures Appl. (9) 36 (1957), 235-249. 
[2] Barbosa, J.L., do Carmo, M.: Stability of minimal surfaces and eigenvalues of the Laplacian . Math. Z. 173 (1980), no. 1, 13-28.

[3] Barbosa, J.L., do Carmo, M., Eschenburg, J.: Stability of hypersurfaces of constant mean curvature in Riemannian manifolds. Math. Z. 197 (1988), no. 1, 123-138.

[4] Barbosa, J.L., Bérard, P.: Eigenvalue and "twisted" eigenvalue problems, applications to CMC surfaces. J. Math. Pures Appl. (9) 79 (2000), no. 5, 427-450.

[5] Chen, B.-Y.:Geometry of Submanifolds. Pure and Applied Mathematics, No. 22. Marcel Dekker, Inc., New York, 1973.

[6] Chen, B.-Y., Yano, K.: Integral Formulas for submanifolds and their applications. J. Diff. Geom. 5 (1971), 467-477.

[7] Cheng, Q-M, Nonaka, K.: Complete submanifolds in Euclidean spaces with parallel mean curvature vector. Manuscripta Math. 105 (2001), 353-366.

[8] Duzaar, F., Fuchs, M.:On the existence of integral currents with prescribed mean curvature vector. Manuscripta Math. 67 (1990), 41-67.

[9] Duzaar, F., Fuchs, M.: On integral currents with constant mean curvature vector. Rend. Sem. Mat. Univ. Padova, 85 (1991), 79-103.

[10] Escobales, R.H.: Riemannian submersions with totally geodesic fibres. J. Differential Geom. 10 (1975), 253-276.

[11] Ferus, D.: On isometric immersions between hyperbolic spaces. Math. Ann. 205 (1973), 193-200.

[12] Freitas, P., Henrot, A.: On the first twisted Dirichlet eigenvalue. Comm. Anal. Geom. 12 (2004), no. 5, 1083-1103.

[13] Frid, H.; Thayer, F.J., An abstract version of the Morse index theorem and its application to hypersurfaces of constant mean curvature. Bol. Soc. Brasil Mat. (N.S.) 20 (1990), no. 2, 59-68.

[14] Gilbarg, D. Trudinger, N.S.:Elliptic partial differential equations of second order, Reprint of the 1998 edition. Classics in Mathematics. Springer-Verlag, Berlin, 2001.

[15] Gulliver, R.:Existence of surfaces with prescribed mean curvature vector, Math. Z. 131 (1973), 117-140.

[16] Gulliver, R.: Necessary conditions for submanifolds and currents with prescribed mean curvature vector, in Seminar on Minimal Submanifolds, Enrico Bombieri, ed., Ann. of Math. Studies 103, Princeton Univ. Press, 1983, pp 225-242.

[17] Harvey, R., Lawson, H.B. Jr.:Calibrated geometries. Acta Math. 148 (1982), 47-157.

[18] Li, G., Salavessa, I.M.C.: Bernstein-Heinz-Chern results in calibrated manifolds. Rev. Mat. Iberoamericana 26(2) (2010), 651-692.

[19] Morgan, F.:Perimeter-minimizing curves and surfaces in $\mathbb{R}^{n}$ enclosing prescribed multivolume. Asian J. Math. 4 no. 2 (2000), 373-383. 
[20] Ranjan, A.:Riemannian submersions of spheres with totally geodesic fibres. Osaka J. Math. 22 (1985), 243-260.

[21] Ritoré, M., Ros, A.: Stable constant mean curvature tori and the isoperimetric problem in three space forms. Comment. Math. Helv. 67 (1992), no. 2, 293-305.

[22] Simons, J.: Minimal varieties in Riemannian manifolds. Ann. Math. 88 (1968) 62-105.

[23] Smale, S.: On the Morse index theorem. J. Math. Mech. 14 (1965), 1049-1055.

[24] Yau, S.T.: Some function-theoretic properties of complete Riemannian manifolds and their applications to geometry. Indiana Math. J. 25 (1976), 659-670. 